\title{
Combining ultra-high-field fMRI adaptation and computational modelling to estimate neuronal frequency selectivity in human auditory cortex
}

\author{
Julien Besle ${ }^{*}$, Rosa-Maria Sánchez-Panchuelo², Susan Francis ${ }^{2}$ and Katrin Krumbholz ${ }^{3}$ \\ 1 Department of Psychology, American University of Beirut, Beirut, Lebanon \\ 2 Sir Peter Mansfield Imaging Centre, School of Physics and Astronomy, University of \\ Nottingham, University Park, Nottingham, NG7 2RD, UK \\ ${ }^{3}$ School of Medicine, University of Nottingham, University Park, Nottingham, NG7 2RD, UK \\ * Corresponding author: julien.besle@aub.edu.lb
}

\section{Abstract}

Frequency selectivity is a ubiquitous property of auditory neurons. Measuring it in human auditory cortex may be crucial for understanding common auditory deficits, but current noninvasive neuroimaging techniques can only measure the aggregate response of large populations of cells, thereby overestimating tuning width. Here we attempted to estimate neuronal frequency tuning in human auditory cortex using a combination of fMRI-adaptation paradigm at $7 T$ and computational modelling. We measured the BOLD response in the auditory cortex of eleven participants to a high frequency $(3.8 \mathrm{kHz})$ probe presented alone or preceded by adaptors at different frequencies ( 0.5 to $3.8 \mathrm{kHz}$ ). From these data, we derived both the response tuning curves (the BOLD response to adaptors alone as a function of adaptor frequency) and adaptation tuning curves (the degree of response suppression to the probe as a function of adaptor frequency, assumed to reflect neuronal tuning) in primary and secondary auditory cortical areas, delineated in each participant. Results suggested the existence of both frequency-independent and frequency-specific adaptation components, with the latter being more frequency-tuned than response tuning curves. Using a computational model of neuronal adaptation and BOLD non-linearity in topographicallyorganized cortex, we demonstrate both that the frequency-specific adaptation component overestimates the underlying neuronal frequency tuning and that frequency-specific and frequency-independent adaptation component cannot easily be disentangled from the adaptation tuning curve. By fitting our model directly to the response and adaptation tuning curves, we derive a range of plausible values for neuronal frequency tuning. Our results suggest that fMRI adaptation is suitable for measuring neuronal frequency tuning properties in human auditory cortex, provided population effects and the non-linearity of BOLD response are taken into account. 


\section{Keywords}

Human; Auditory cortex; fMRl; Adaptation; Frequency selectivity

\section{Introduction}

Frequency specificity is a fundamental organizing principle of the auditory system and accurate frequency representation is crucial for auditory perception. Many auditory neurons are tuned to a limited range of frequencies. Animal work has suggested that frequency tuning changes from the cochlea to the cortex, but there is no consensus yet as to whether cortical frequency tuning is broader or finer than cochlear frequency tuning [1, 2]. In humans, cochlear frequency tuning can be estimated either behaviourally, using notched-noise masking, or from otoacoustic emission latencies [3]. However, there is currently no accepted measure of neuronal frequency tuning in the human auditory cortex. Here, we combine an fMRI adaptation paradigm with computational modelling to estimate cortical neuronal tuning widths non-invasively in normal-hearing participants.

Previous fMRI studies have estimated population frequency tuning properties in human auditory cortex by measuring voxelwise blood oxygen level-dependent (BOLD) responses to different frequencies [4-7]. Whilst these voxelwise population receptive field estimates are useful, for instance to locate primary (or "core") auditory areas, thought to have narrower frequency tuning than surrounding secondary (or "belt") areas [4, 7], they reflect the aggregate tuning properties of tens to hundreds of thousands of neurons contained within a given voxel $[8,9]$, and thus probably overestimate the tuning width of individual neurons. The spatial spread of the BOLD response beyond the neurally active area [10] likely aggravates this problem further. It has been suggested that neuronal tuning properties can instead be probed through adaptation, or repetition suppression. In the simplest adaptation paradigm, the adaptor-probe paradigm pioneered in psychophysical studies [11], the response to a probe stimulus is measured when preceded either by an identical adaptor stimulus or by adaptors differing from the probe on a given stimulus feature. The decrease in probe response (adaptation) as a function of the similarity between the adaptor and probe is then used to infer neuronal tuning properties with respect to the relevant feature: if the measured population contains neurons selective for the feature, adaptation will be greater when the adaptor is identical to the probe than when it is different, and the tuning of the adaptation effect at the population level is thought to reflect the specificity of neuronal tuning $[12,13]$. Adaption paradigms have been widely used in visual fMRI studies to probe neuronal tuning properties to features such as orientation and motion direction in different cortical visual areas [e.g. 14, 15]. In the auditory domain, adaptation paradigms have similarly been used in EEG studies to measure neuronal frequency tuning through adaptation of the $\mathrm{N} 1$ and P2 deflections of the cortical auditory-evoked potentials [16-21]. However, because the N1 and 
P2 comprise contributions from multiple cortical areas $[22,23]$, the exact cortical origin of the observed frequency tuning remains unclear.

The current study aimed to test whether adaptation can be used in conjunction with fMRI to infer neuronal frequency selectivity, or tuning width, within auditory cortex. Using the adaptorprobe paradigm, we derive both frequency response tuning curves (BOLD responses to adaptors at different frequencies) and frequency adaptation tuning curves (BOLD response suppression to probes preceded by different adaptors), in multiple auditory cortical areas that were delineated in individual participants based on tonotopic gradient reversals [4]. We then use a computational model of a tonotopically organized cortical sheet to relate these population frequency tuning curves to the underlying neuronal tuning and adaptation properties, as well as the non-linear properties of the BOLD response. By jointly fitting the model to the response and adaptation tuning curves, we derive a range of plausible neuronal tuning width estimates for both primary and non-primary auditory cortical areas.

\section{Results}

\section{FMRI adaptation in the human core and belt auditory cortex is} frequency-specific

To investigate the frequency-specificity of $f M R I$ adaptation, we measured BOLD responses to adaptor and probe stimuli presented in isolation ( $A$ and $P$ responses) or as adaptor-probe pairs (AP response). The adaptor frequency varied between 0.251 and $6.009 \mathrm{kHz}$ for $\mathrm{A}$, and between 0.505 and $3.839 \mathrm{kHz}$ for $\mathrm{AP}$, whilst the probe frequency was fixed at $3.839 \mathrm{kHz}$ (Fig. 1).

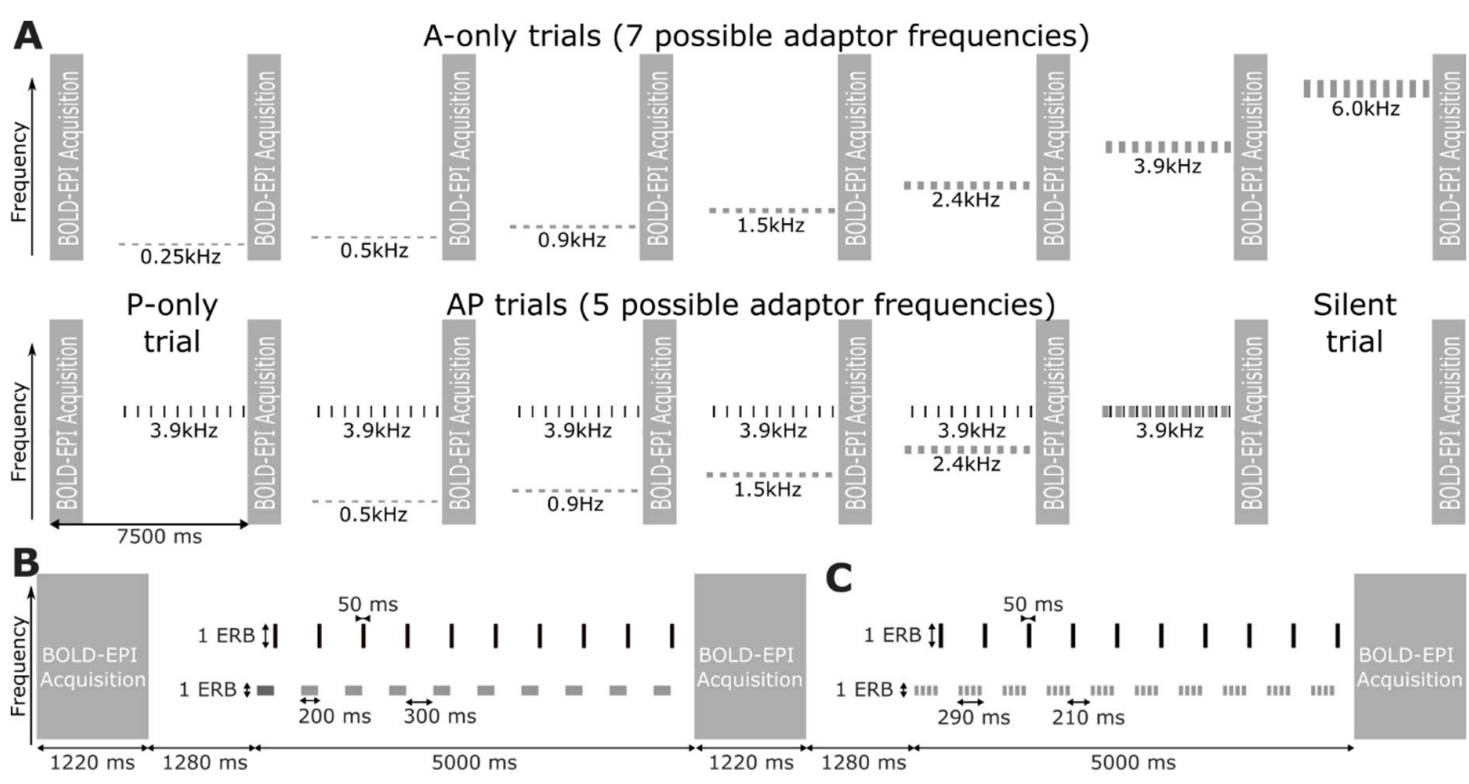

Figure1: Stimulus presentation and fMRI acquisition timing. A. Timelime of 15 example fMRI trials showing all seven adaptor (A) trials, all five adaptor + probe (AP) trials, one probe (P) trial and one null (silent) trial. The long vertical bars represent the fMRI volume acquisitions. The short 
bars show the stimulus trains presented in between acquisitions, with center frequency and tuning width indicated on the vertical axis (representing a linear frequency scale). B\&C. Detailed timeline of a single AP trial presented to the first five (left) and last six (right) participants, respectively. A and $P$ trials followed the same timeline, with either the probes or the adaptors removed from the train.

We used the responses to the seven adaptors presented alone $(A)$ to derive tonotopic maps in each hemisphere of each participant and defined four regions of interest corresponding to the core and belt auditory cortex (Fig. 2). Two ROls in and around Heschl's gyrus corresponded to two mirror-reversed tonotopic gradients overlapping with the core (or primary) auditory cortex, as marked by intracortical myelin and response tuning measurements: the anterior gradient ROI [overlapping most with the core;4] and the posterior gradient ROI. The other two ROIs, henceforth referred to as anterior and poster belt ROls comprised all auditory-responsive voxels located either anteriorly or posteriorly to the gradient ROls.

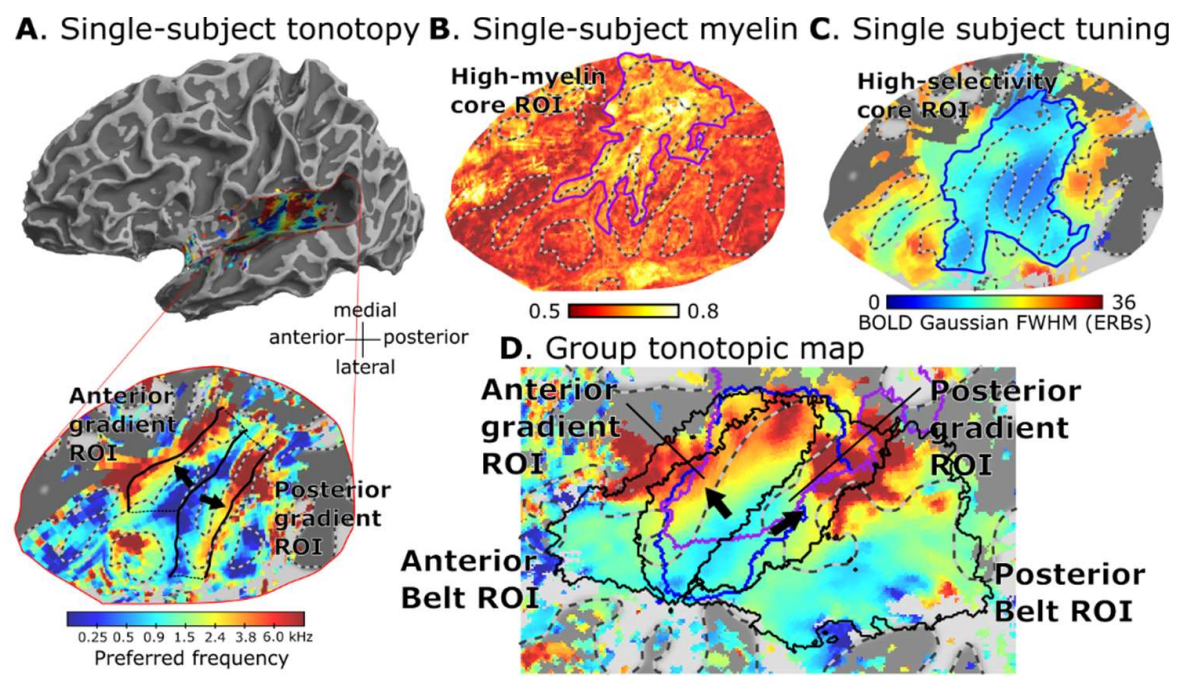

Figure 2: ROI definition at individual participant level and resulting probabilistic ROI location on group template. A. Map of voxelwise preferred-frequency map on semi-inflated (top row) and flattened (bottom row) cortical surface reconstructions of the left hemisphere and auditory cortex for participant 2. Preferred frequencies are shown on a quasi-logarithmic color scale (representing the cochlear frequency scale, see Material and Methods). Thick black solid lines show automatically-detected reversals in tonotopic gradients that were used to define the anterior and posterior gradient ROIs. Thin dotted lines join the reversals between the 2 gradient ROIs. Black arrows indicated the average direction of the tonotopic gradient within each ROI. Gyri are indicated by light-gray, and sulci by dark-gray highlight. The borders between them are shown by two-tone gray dashed lines. B. Individual map of PSIR-derived intracortical myelin content, proportional to the longitudinal relaxation rate R1. The purple outline show a region of high myelination, thresholded an individualized criterion [4]. C. Individual map of voxelwise BOLD response tuning width, measured as FWHM in cochlear ERBs. The blue outline highlights a region of high frequency selectivity (low tuning width). D. Group preferred-frequency map obtained by spherical deconvolution of the 22 individual hemispheres. Black, purple and blue outlines show the average location of the individual ROls shown in A-C across the 22 hemispheres, based on the maximum probability value of each ROI at each voxel of the template cortical surface (see Methods for details).

To estimate the adaptation effect of a given adaptor, $A$, on the response to the probe, $P$, we first subtracted A from AP (for the same adaptor frequency) to obtain the adapted probe 
response, $\mathrm{P} \mid \mathrm{A}$, and we then computed the adaptation effect by taking the difference between the unadapted and adapted probe responses $P$ and $P \mid A$. Figure $3 A-E$ shows groupaverage maps of the adaptation effect for increasing adaptor frequencies. There were significant adaptation effects for all adaptor frequencies but, as expected, the adaptation effect increased in amplitude as the adaptor frequency approached the probe frequency. When the adaptor and probe frequencies were similar or equal (Fig. 3C-E), the adaptation effect was largest at the cortical locations of the two high-frequency tonotopic reversals separating each of the two gradient ROls from the adjoining belt ROI (also corresponding to the locations of largest response to the probe, which had relatively high frequency). Significant adaptation was also observed in the remainder of the posterior belt $\mathrm{ROI}$ at all adaptor frequencies and this adaptation effect seemed to depend less on the adaptor frequency (i.e. appeared somewhat frequency-independent). Finally, despite adaptation being strongest in high-frequency-preferring regions (because the probe itself was high frequency), maximum adaption (i.e. when the adaptor and probe frequencies matched) was observed to be approximately constant when expressed as a proportion of the unadapted probe response [(P-A |P)/P, Fig. 3F], at around 75\% throughout auditory cortex.

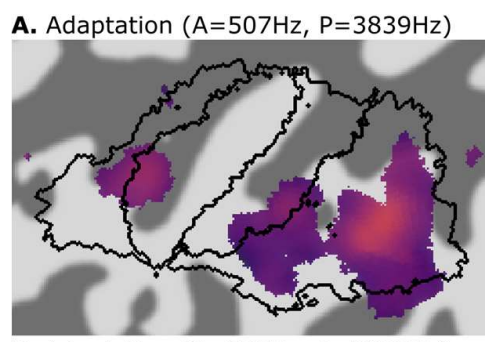

D. Adaptation $(A=2423 \mathrm{~Hz}, P=3839 \mathrm{~Hz})$
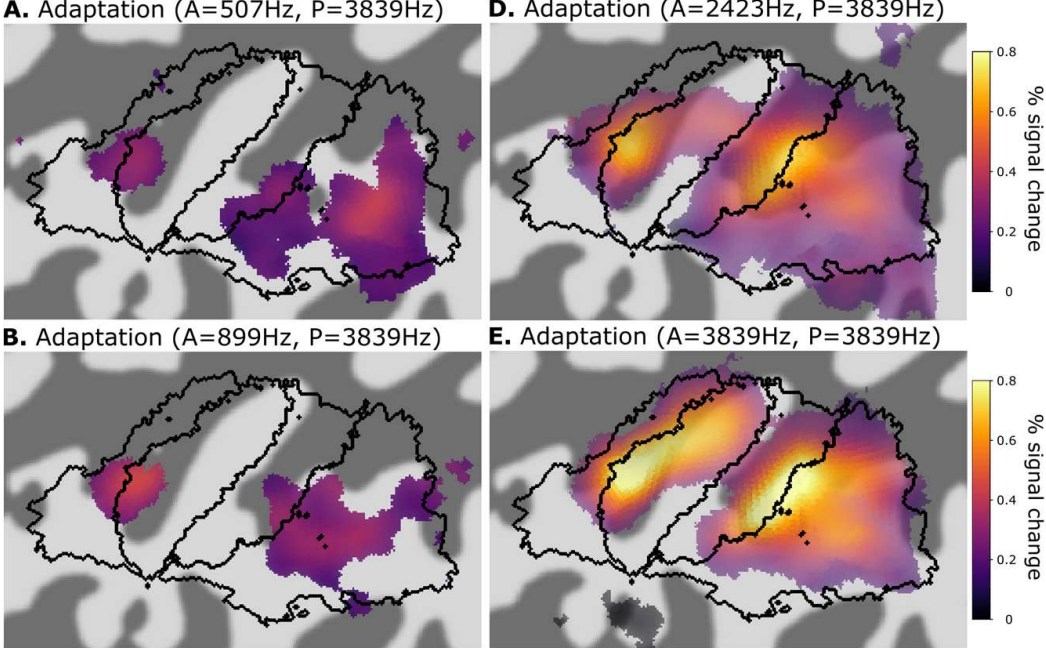

E. Adaptation $(A=3839 \mathrm{~Hz}, P=3839 \mathrm{~Hz}$

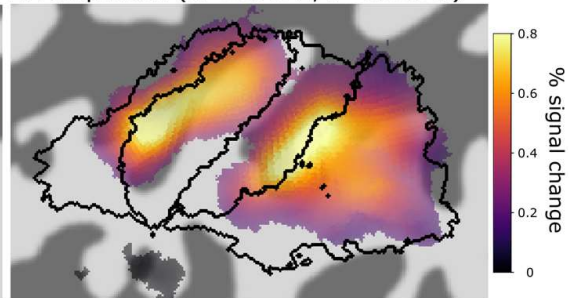

C. Adaptation $(A=1501 \mathrm{~Hz}, \mathrm{P}=3839 \mathrm{~Hz})$ F. Normalized adaptation $(A=3839 \mathrm{~Hz}, \mathrm{P}=3839 \mathrm{~Hz})$

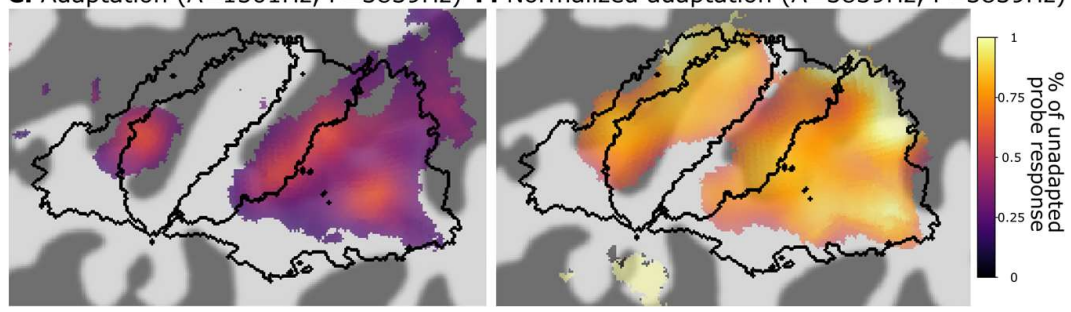

Figure 3: Group-level maps of the frequency adaptation effect for different adaptor-probe frequency differences. A-E. Amplitude of the adaptation effect $[P-P \mid A=P-(A P-A)=A+P-$ AP] for adaptors ranging between 507 and $3839 \mathrm{~Hz}$ and a $3839 \mathrm{~Hz}$ probe. Black outlines show the approximate location of ROls (based on maximum probabilities, see Fig. 2C). Maps are, smoothed along the cortical surface (FWHM=6mm), thresholded at $p<0.05$ FDR-corrected and transparency is a power function of $-\log 10$ (p). F. Same as $E$, but voxelwise adaptation values have been normalized by the corresponding voxelwise probe response $[(P-P \mid A) / P]$. 
We constructed frequency adaptation tuning curves by plotting the ROI-average adaptation effects as a function of the adaptor frequency (expressed in human cochlear frequency units or ERBS). Figure $4 \mathrm{~A}$ shows the ROI-averaged unadapted $(\mathrm{P})$ and adapted $(\mathrm{P} \mid \mathrm{A})$ probe responses and Figure $4 \mathrm{~B}$ shows the normalized ROI-average adaptation tuning curves [(P$A|P| / P]$ for each of the four (two gradient and two belt) ROls. Confirming our observations on the group-average maps, adaptation tuning curves were frequency-tuned in all four ROls, with adaptation increasing with decreasing adaptor-probe frequency difference. The maximum adaptation effects (when the adaptor and probe frequencies were equal) were similar across ROIs, ranging between 63 and $74 \%$. The minimum adaptation effect, on the other hand, was smaller for the gradient ROIs ( 10\%) and larger for the belt ROls ( 35\%). In all ROls, the adaptation tuning curves seemed to reach a non-zero asymptote towards large adaptorprobe frequency differences, suggesting a possible constant, or frequency-unspecific, baseline adaptation.
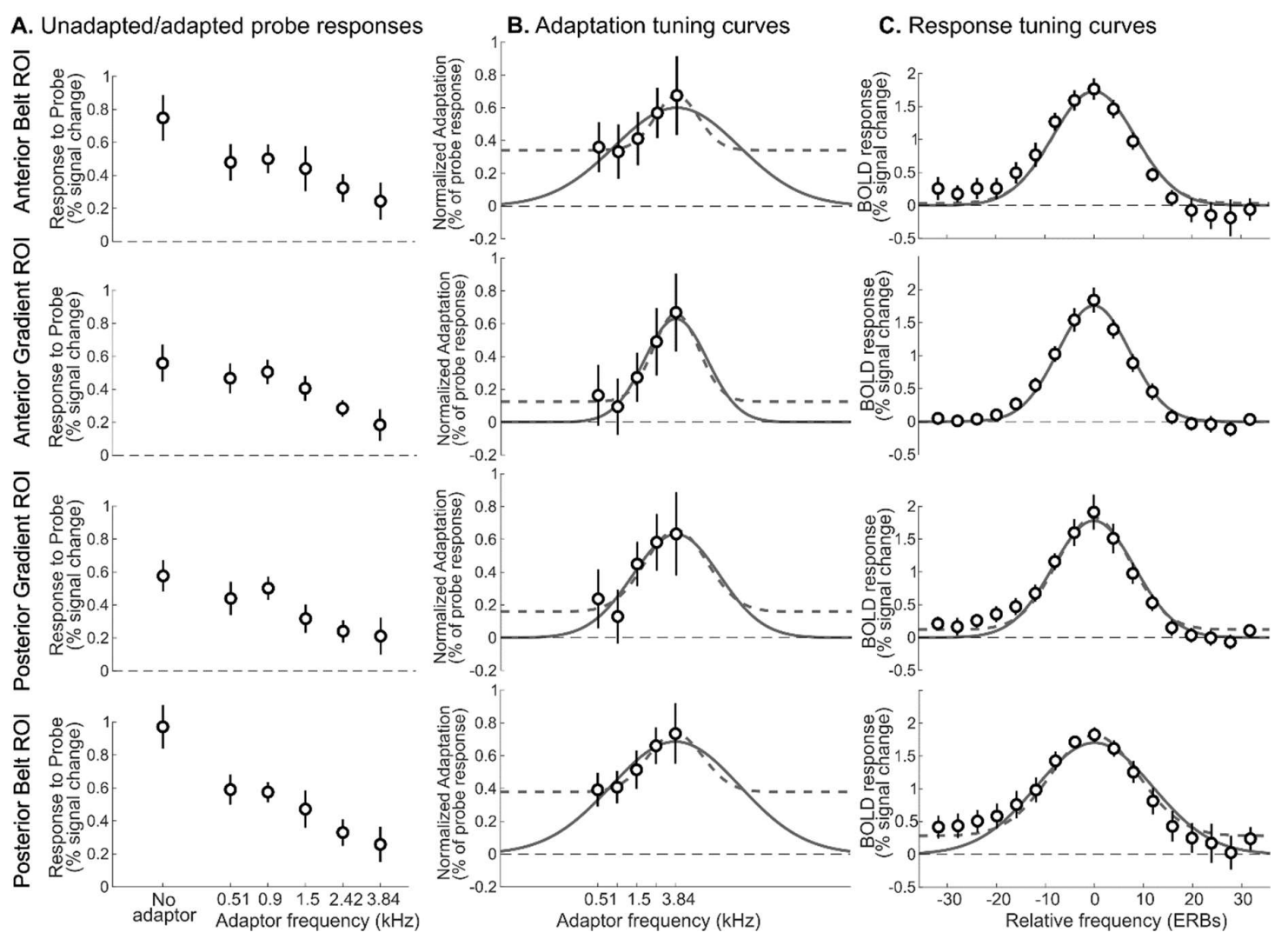

Figure 4: $B O L D$ adaption and $B O L D$ response tuning curves in core and belt region of auditory cortex. A. Unadapted (P) and estimated adapted (AP-A) BOLD responses to the probe in the two gradient ( $\approx$ core) and the two belt ROls, averaged across participant and hemispheres. Adaptor frequencies on the $X$ axis are equally spaced on the cochlear ERB-number scale, but expressed in $\mathrm{kHz}$. B. ROl-average BOLD adaptation effect expressed as percent decrease from the unadapted response to the probe $[(P-P \mid A) / P]$ as a function of the adaptor's center frequency (i.e. BOLD adaptation tuning curve). The solid gray curve is and the dashed gray curve is the best-fitting Gaussian including a frequency-independent adaptation offset (see Fig. 5 and Table 1 for best-fitting parameters). C. ROl-average voxelwise BOLD response to 
adaptors alone (A), as a function of the frequency difference between the A stimulus and the preferred frequency of each voxel (i.e. BOLD response tuning curve). Frequency differences on the $X$ axis are expressed in ERBs. Solid and dashed gray curves as in panel B. In all panels, error bars represent $95 \%$ bootstrap confidence intervals.

To estimate the tuning width of the adaptation effect, we fitted the adaptation tuning curves with two different types of Gaussian-shaped functions centered on the probe frequency (solid lines in Fig. 4B). For the first fit, we assumed that adaptation was entirely frequency-specific, and thus that the adaptation effect would approach zero for infinitely large adaptor-probe frequency differences. The resulting adaptation tuning widths (solid back traces in Fig. 5, see also Table 1) were smallest in the anterior gradient ROI (13.9 ERBs, or $\sim 2.3$ oct), slightly wider in the posterior gradient ROI (16.4 ERBs, or $\sim 2.7 \mathrm{oct}$ ) and widest in the anterior and posterior belt ROIs (36.6 and 38.4 ERBs, or $\sim 6$ oct; main effect of ROI: $F(3,50.02)=5.6826, p=0.0020$; all pairwise comparisons significant except between the two belt ROI and the two gradient ROIs). By not considering a possible frequency-independent adaptation component, this first fit may, however, have overestimated the tuning widths of the frequency-specific component. Hence, for the second fit, we instead assumed that adaptation could reach a non-zero asymptote for infinite adaptor-probe frequency differences (modelled as a constant offset; see dashed lines in Fig. 4B). The best-fitting asymptotic adaptation values ranged between 8 and $35 \%$ for different ROIs (Table 1; significantly different from 0 in both belt ROIs, $p<.0005$, but not in either gradient ROI) and were significantly smaller in the gradient than in the belt ROls (main effect of $R O I: F(3,50.02)=14.482, p=7 \times 10^{-7}$; all pairwise comparisons significant except between the two belt ROI and the two gradient ROls). The second fit also yielded substantially narrower frequency-specific adaptation tuning width estimates (dashed black lines in Fig. 5, see also Table 1), ranging between 10.4 and 13.6 ERBs across ROls, and with no significant differences across ROIs $(F(3,48.5)=0.4464, p=0.72)$. Figure $4 B$ shows that the second fit yielded $a$ consistently better match to the data across all ROIs (also shown by larger r-squared values; see Table 1). However, due to the large variance in adaptation values between participants and the limited range of adaptor frequencies, this improvement in model fit did not lead to a similar increase in model evidence quantified through the Bayesian information criteria (BIC), which instead favored the first fit(see Table 1). Maps of voxelwise (or searchlight-) fitted Gaussian parameters, both with and without frequency-independent adaptation offset are shown in supplementary Figure 1. 


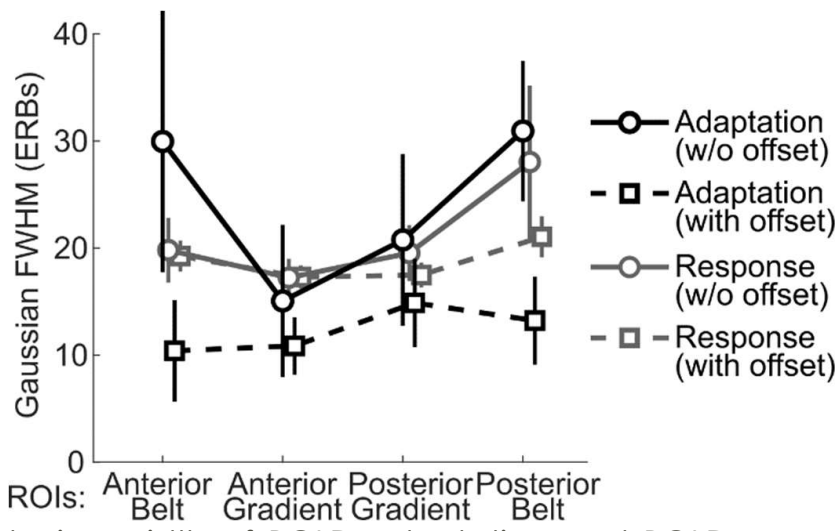

Figure 5: Estimated tuning width of BOLD adaptation and BOLD response tuning curves in gradient (core) and belt ROls (black = adaptation; gray = response). Tuning width was estimated by fitting Gaussians to the group-average tuning curves, with or without frequencyindependent adaptation offsets (dashed and solid traces respectively). See Table 1 for all other best-fitting Gaussian parameters. Error bars represent $95 \%$ bootstrap confidence intervals.

\begin{tabular}{|c|c|c|c|c|c|c|c|c|c|}
\hline & \multicolumn{9}{|c|}{ BOLD adaptation tuning width (Simple Gaussian) } \\
\hline & FWHM (ERBs) & FWHM (oct.) & Scaling & $\triangle \mathrm{BIC}$ & $\triangle \mathrm{AlCc}$ & $\mathrm{BIC}$ & $\mathrm{AlCc}$ & $r^{2}$ & Outliers \\
\hline Anterior Belt & $36.58 \pm 12.37$ & $6.07 \pm 2.04$ & $0.74 \pm 0.18$ & 2.2 & -0.4 & -172.6 & 134.3 & 0.063 & 4 \\
\hline Anterior gradient & $13.87 \pm 6.88$ & $2.28 \pm 1.13$ & $0.74 \pm 0.19$ & 3.0 & 0.4 & -158.4 & 148.5 & 0.159 & 3 \\
\hline Posterior Gradient & $16.36 \pm 8.02$ & $2.70 \pm 1.32$ & $0.75 \pm 0.20$ & 3.9 & 1.3 & -159.1 & 147.8 & 0.129 & 3 \\
\hline \multirow[t]{3}{*}{ Posterior Belt } & $38.40 \pm 7.08$ & $6.37 \pm 1.17$ & $0.74 \pm 0.15$ & 2.1 & -0.5 & -246.9 & 60.0 & 0.142 & 2 \\
\hline & \multicolumn{9}{|c|}{ BOLD adaptation tuning width (Gaussian with offset) } \\
\hline & FWHM (ERBs) & FWHM (oct.) & Scaling & & & $\mathrm{BIC}$ & $\mathrm{AlCc}$ & $r^{2}$ & Outliers \\
\hline Anterior Belt & $10.37 \pm 4.54$ & $1.71 \pm 0.75$ & $0.45 \pm 0.17$ & 0.30 & 0.15 & -170.4 & 133.86 & 0.084 & 0 \\
\hline Anterior gradient & $11.73 \pm 2.72$ & $1.93 \pm 0.45$ & $0.63 \pm 0.18$ & 0.08 & 0.16 & -155.4 & 148.9 & 0.172 & 0 \\
\hline Posterior Gradient & $13.59 \pm 4.07$ & $2.24 \pm 0.67$ & $0.63 \pm 0.21$ & 0.12 & 0.17 & -155.2 & 149.1 & 0.136 & 0 \\
\hline \multirow[t]{3}{*}{ Posterior Belt } & $13.03 \pm 4.27$ & $2.15 \pm 0.70$ & $0.44 \pm 0.14$ & 0.35 & 0.10 & -244.8 & 59.472 & 0.163 & 0 \\
\hline & \multicolumn{9}{|c|}{ BOLD response tuning width (Simple Gaussian) } \\
\hline & FWHM (ERBs) & FWHM (oct.) & Scaling & $\triangle \mathrm{BIC}$ & $\triangle \mathrm{AICC}$ & $\mathrm{BIC}$ & $\mathrm{AlCc}$ & $r^{2}$ & Outliers \\
\hline Anterior Belt & $22.85 \pm 3.20$ & $4.13 \pm 0.57$ & $1.73 \pm 0.19$ & 5.2 & 1.3 & -616.3 & 437.23 & 0.658 & 0 \\
\hline Anterior gradient & $17.99 \pm 1.83$ & $3.24 \pm 0.33$ & $1.77 \pm 0.18$ & 5.9 & 2.0 & -897.3 & 156.22 & 0.807 & 0 \\
\hline Posterior Gradient & $21.35 \pm 2.47$ & $3.85 \pm 0.44$ & $1.80 \pm 0.26$ & -8.2 & -12.1 & -709.6 & 343.92 & 0.702 & 0 \\
\hline \multirow[t]{3}{*}{ Posterior Belt } & $31.25 \pm 6.84$ & $5.69 \pm 1.22$ & $1.76 \pm 0.13$ & -19.3 & -23.2 & -510.9 & 542.67 & 0.531 & 0 \\
\hline & \multicolumn{9}{|c|}{ BOLD response tuning width (Gaussian with offset) } \\
\hline & FWHM (ERBs) & FWHM (oct.) & Scaling & & Offset & $\mathrm{BIC}$ & $\mathrm{AlCc}$ & $r^{2}$ & Outliers \\
\hline Anterior (belt) & $19.59 \pm 1.43$ & $3.53 \pm 0.26$ & $1.74 \pm 0.25$ & 0.02 & 0.15 & -611.2 & 438.5 & 0.658 & 0 \\
\hline Myelin-derived core & $17.44 \pm 1.06$ & $3.14 \pm 0.19$ & $1.78 \pm 0.20$ & -0.01 & 0.07 & -891.5 & 158.2 & 0.807 & 0 \\
\hline Tuning-derived core & $18.02 \pm 1.13$ & $3.24 \pm 0.20$ & $1.74 \pm 0.26$ & 0.11 & 0.08 & -717.8 & 331.9 & 0.713 & 0 \\
\hline Posterior (belt) & $22.06 \pm 1.82$ & $3.98 \pm 0.33$ & $1.59 \pm 0.20$ & 0.26 & 0.16 & -530.2 & 519.5 & 0.561 & 0 \\
\hline
\end{tabular}

Table 1: Average parameter estimates for the Gaussian function fits of the ROl-average adaptation and response tuning curves, without and with a frequency-independent component (offset parameter). Averages are given with their $95 \%$ bootstrap confidence intervals. The fitted function widths are given as half-maximum widths (FWHM) in both ERBs and transformed to approximate octave values (w.r. to the center of the adaptor frequency range for response tuning curves and to the probe frequency for adaptation tuning curves). The scale parameter refers to the best-fitting peak response in percent signal change for the response tuning curves, or the maximum normalized adaptation amount in percent for the adaptation tuning curves. For the second of the tuning curve fits, the offset parameter gives 
the best-fitting amount of frequency-independent adaptation/response, in the same units as the scaling parameter. The rightmost column in each table shows the number of rejected outliers for computing group-average parameters and for the ANOVAs testing for differences between ROls. BIC: Bayesian Information criterion, AICc: Akaike information criterion, corrected for small sample size. $\triangle \mathrm{BIC}$ and $\triangle \mathrm{AICC}$ give the differences in $\mathrm{AICC}$ and $\mathrm{BIC}$ between the Gaussian model with and without offset. Positive values indicate evidence for the simpler model (without offset).

\section{Comparison between $\mathrm{fMRI}$ adaptation and BOLD response tuning} curves

Under the assumption that fMRI adaptation reflects underlying neuronal tuning properties (see Introduction), our fMRI adaptation tuning curves would be expected to be narrower than the response tuning curves based on the BOLD responses to the adaptor-alone stimuli. To construct ROI-average response tuning curves for direct comparison with the respective adaptation tuning curves, we averaged the voxelwise adaptor-alone responses after centering them on their respective preferred frequencies (to avoid averaging tuning curves with different maxima, see Methods section). Figure $4 \mathrm{C}$ shows that the ROI-average response tuning curves were approximately Gaussian-shaped when frequency was expressed in ERB-number (or cochlear frequency) units. Thus, to measure their widths, we fitted them with Gaussian functions. As for the adaptation tuning curves, we used Gaussians both with and with a constant offset. The resulting tuning widths estimates (solid and dashed gray curves in Fig. 5) were narrowest in the anterior gradient ROI, with an average of 18.0/17.4 ERBs (w/wo offset), corresponding to $\sim 3.2 / 3.1$ octaves (see Table 1), and increased anteriorly and posteriorly towards the belt regions (first fit: $F(3,63)=15.055, p=2 \times 10^{-7}$; second fit: $F(3,63)=13.566, p=6 \times 10^{-}$ 7). The tuning width estimates from the first and second fits were generally similar, except for the posterior belt ROI, for which the first fit yielded a substantially greater tuning width than the second fit. Only the posterior belt ROI showed a statistically significant response offset $(p=$ 0.0003; see Fig. 4C), but most ROls (except the anterior gradient) showed an asymmetric asymptotic offset, larger at frequencies below than above the tuning curve peak.

When fitting Gaussian fits without constant offset, the response tuning widths were broadly similar to the corresponding adaptation tuning widths (see Fig. 5) - contrary to the expectation that they reflect neuronal tuning properties. In fact, in the anterior belt ROI, which also showed a large degree of asymptotic adaptation, the adaptation tuning width was considerably larger (although not significantly) than the response tuning width. In contrast, the second Gaussian fits (with offset) were consistent with the idea that adaptation tuning reflects neuronal tuning, in that adaptation tuning widths were smaller than response tuning widths across all ROls. The differences were larger in the belt ROls, where the response tuning widths were nearly twice as large as the adaptation tuning widths, than in the gradient ROls (interaction between $\mathrm{ROI}$ and tuning curve type: $\mathrm{F}(3,109.56)=4.08, \mathrm{p}=0.009$; comparisons between response and adaptation tuning widths significant at $p<0.03$ in all ROIs). 


\section{Using a computational model to explore the quantitative relationships between $\mathrm{fMRI}$ adaptation, BOLD response and neuronal tuning} So far, our results suggest that fMRI adaptation tuning curves may have two components: a frequency-independent component (the asymptotic offset of the adaptation tuning curve), and a frequency-tuned component with narrower frequency tuning than the BOLD responses that may reflect the underlying neuronal frequency tuning. To better understand how fMRI adaptation and response tuning quantitatively relate to the underlying neuronal tuning properties, we here use a simple computational model of fMRI adaption in a unidimensional cortical strip of frequency-tuned neurons (illustrated in Fig. 6A,E). The model neurons had Gaussian-shaped frequency tuning functions (on the ERB-number scale) with nominal characteristic frequencies increasing monotonically from one side of the array to the other. The response tuning width of individual auditory neurons, $\sigma$, was assumed to be constant (in ERBs) and identical for all neurons. While the nominal characteristic frequency of neurons increased tonotopically, their actual characteristic frequency at a given location within the array was allowed to randomly vary according to a Gaussian distribution of width $\delta$, representing the tonotopic scatter, which controlled the degree of tonotopic order. The model implemented adaptation both at the neuronal level and at the voxel level [reflecting BOLD nonlinearity in the latter case, see e.g. 36]. Neuronal adaptation was implemented as a simple multiplicative suppression, with the suppression factor proportional to the size of the neuron's response to the adaptor. The maximum amount of neuronal adaptation (when the adaptor and probe frequencies were equal) was controlled by a proportionality parameter, $\kappa$. BOLD non-linearity was implemented through a compressive sigmoid function, which was applied to the aggregate response of all neurons contained within a given voxel. The saturation parameter $\gamma$ controlled the maximum possible BOLD response and thus the degree of compression (see Methods section for more implementation details).

To informally test the behavior of the model and its ability to reproduce our experimental results in response to adaptor and probe stimuli of varying frequencies, we manually set the four above free parameters $(\sigma, \delta, \kappa$ and $\gamma)$. Across all informal tests, we set the neuronal tuning parameter $\sigma$ to 5 ERBs (shown in Fig. 6B) - close to the average cellular tuning width reported for non-human primates (see Discussion section). 

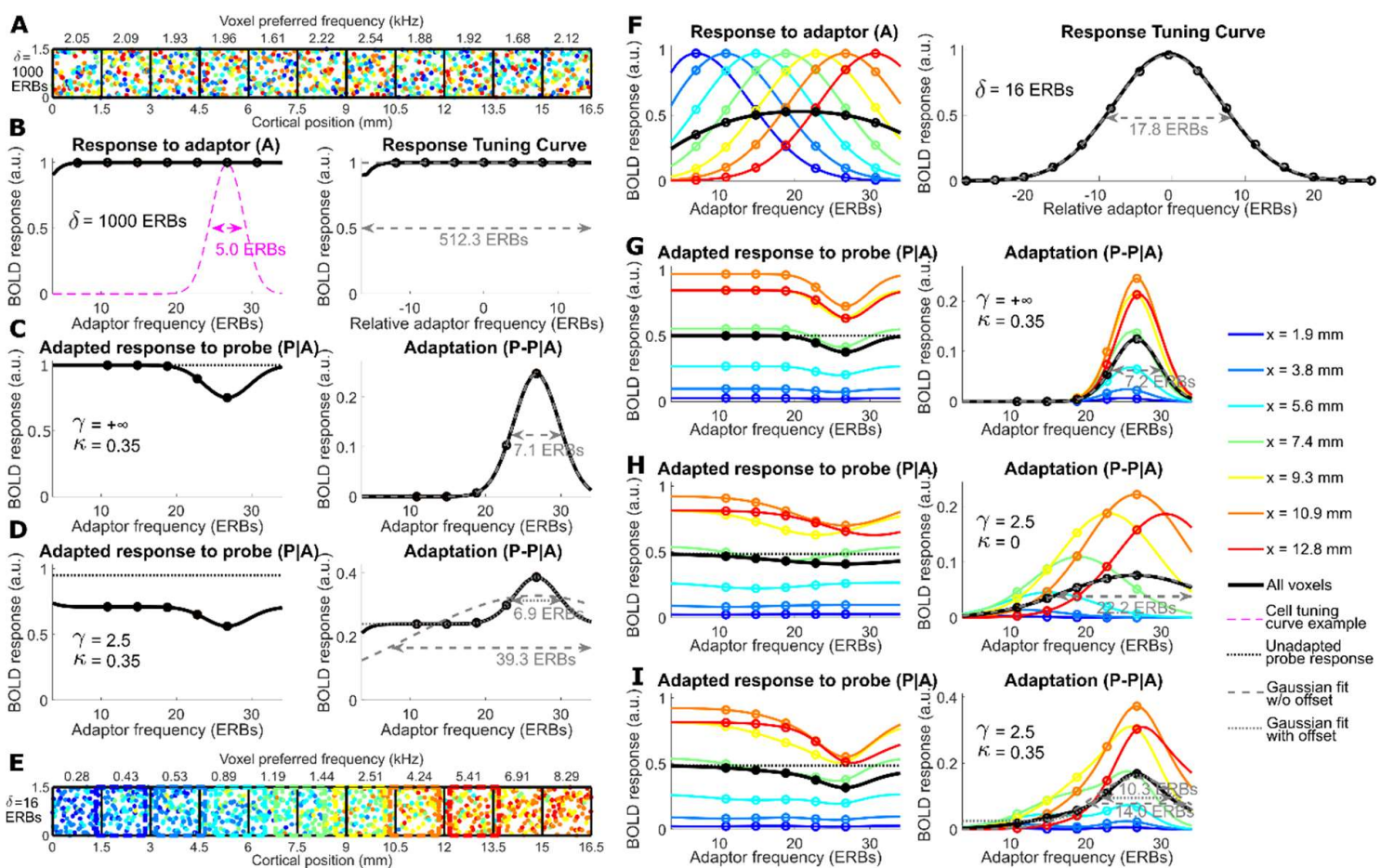

$\begin{aligned} \mathrm{x} & =7.4 \mathrm{~mm} \\ \mathrm{x} & =9.3 \mathrm{~mm}\end{aligned}$

I Adapted response to probe (PIA)

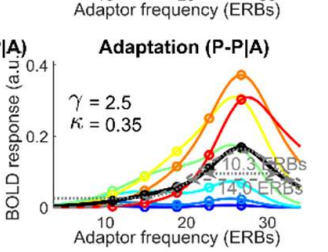

$\mathrm{x}=10.9 \mathrm{~mm}$

- $\mathrm{x}=12.8 \mathrm{~mm}$

-All voxels

- Cell tuning

......... - Unadapted

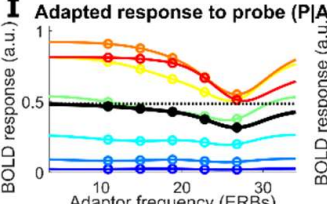

- - Gaussian fit

......... Gaussian fit

Figure 6: Simulated BOLD response and BOLD adaption tuning curves in a cortical model of fMRI adaptation for different tonotopic scatter, cell adaptation strength and BOLD saturation parameters. A. Graphical illustration of the spatial layout of the model without tonotopic organization (tonotopic scatter $\delta=1000$ ERBs). The black grid represents contiguous voxels extending the full length of the model and the colored dots represent the spatial cortical position of 100 randomly-drawn cells per voxel, with the color of the dot coding for best frequency (blue = low frequency and red = high frequency). The actual model also included intermediate voxels overlapping with the represented voxels. Figures above each voxel give the estimated preferred frequency of the voxel. Although cells and voxels are represented here in 2D for clarity, the actual model was unidimensional (the represented transverse dimension was collapsed). Because of the lack of tonotopic organization, the modelled BOLD response was identical at all of voxels and is illustrated in panels B to D. B. Modelled voxel BOLD responses to adaptor-only trials $(A)$ as a function of adaptor frequency (left panel) and BOLD response tuning curve (right panel) obtained by averaging voxel tuning curves re-centered on their respective center frequency. In both panels, open dots correspond to the 7 adaptor frequencies actually used in our fMRI experiment, while black traces show responses with a better frequency resolution (50 adaptor frequencies between 0.19 and $7.82 \mathrm{kHz}$ ). In the left panel, the response to the $3.84 \mathrm{kHz}$ adaptor also corresponds to the unadapted response to the probe $(P)$. The dashed magenta trace illustrates the tuning curve of an auditory cell responding maximally to the probe and a tuning width of 5 ERBs FWHM. The modelled BOLD response tuning curve (right panel) was fitted with a Gaussian (gray dashed line) whose FWHM (gray double-arrow) is reported in ERBs. C. Adapted BOLD response to the probe as a function of adaptor frequency (left panel) and unnormalized BOLD adaptation tuning curve (right panel) computed by subtracting the adapted probe response from the unadapted probe response (replotted from the left panel of $B$ as a horizontal dotted line), for an instance of the model including neuronal adaptation ( $\kappa=0.35$ ) but no BOLD non-linearity (BOLD saturation parameter $\gamma=+\infty)$. D. Same as C, but the BOLD saturation $\gamma$ was set to 2.5. The BOLD adaptation tuning curve (right panel) was fitted both with a simple Gaussian (gray dashed line) and a Gaussian with an offset parameter (gray dotted line). Corresponding Gaussian FWHM are reported below the double arrows. E. Same as A, but the tonotopic scatter was reduced to $\delta=16$ ERBs, resulting in a tonotopically-organized model. The modelled BOLD responses of the 7 voxels outlined with colored dashed lines are illustrated in panels $F$ to $I$ in the corresponding color. F. Same as B with $\delta=16$ ERBs instead of 1000 ERBs. G. Same as C, but with 
$\delta=16$ ERBs instead of 1000 ERBs. H. Same as G, but with BOLD non-linearity ( $\gamma=2.5$ as in D) and without cell adaptation $(\kappa=0)$. I. Same as $G$ and $\mathrm{H}$, but with both cell adaption $(\kappa=0.35)$ and BOLD non-linearity $(\gamma=2.5)$.

We first tested a version of the model without tonotopic organization (by setting $\delta$ to a large number - 1000 ERBs). In this version, neuronal characteristic frequencies were essentially uniformly distributed across the array (Fig. 6A), and thus the BOLD response tuning curves (response to A-only stimuli) were essentially flat, both voxelwise and on average across all voxels (Fig. 6B), whether or not the voxelwise response tuning curves were re-centered on their preferred frequencies before averaging (compare left and right panels in Fig. 6B). Figure 6C (left panel) shows voxelwise and array-average BOLD responses to the $3839-\mathrm{Hz}$ probe when preceded by different adaptor frequencies when only neural adaptation was at play $(\kappa=0.35)$ and there was no BOLD non-linearity $(\gamma=+\infty)$. In this (unrealistic) scenario, the BOLD response to the probe only decreased for adaptor frequencies close to the probe frequency, resulting in a narrowly tuned $\mathrm{fMRI}$ adaptation tuning curve (right panel). The obtained BOLD adaptation tuning width (7.1 ERBS FWHM) was nevertheless slightly larger than the underlying 5-ERB neuronal tuning width (by a factor $\sqrt{2}$, see Discussion). In this non-tonotopic model, introducing BOLD non-linearity (by setting $\gamma$ to 2.5 ) resulted in an additional, general reduction of the adapted probe responses (Fig. 6D, left panel) and a corresponding frequency-independent adaptation component in the array-average fMRI adaptation tuning curve (Fig. 6D, right panel). Fitting this adaptation tuning curve with a simple Gaussian, without constant offset, yielded a tuning width of 39.3 ERBs - more than an order of magnitude larger than the underlying 5-ERB neuronal tuning width. However, fitting a Gaussian with an added offset yielded an adaptation tuning width of 6.9 ERBs - similar to the adaptation tuning width in the absence of BOLD non-linearity (7.1 ERBs).

The above simulations demonstrate that, for non-topographically-organized neuron populations, the adaptive effect of neuronal adaptation and BOLD non-linearity are easily distinguishable, because the former is stimulus-specific, whilst the latter is stimulus-independent. This ceased to be the case, however, when the degree of tonotopic order was increased (by decreasing the scatter parameter $\delta$ to 16 ERBs FWHM), such that neuronal characteristic frequencies increased on average across voxels (Fig. 6E). In this case, the voxelwise BOLD response tuning curves (Fig. 6F, left panel) were no longer flat but became peaked, with maximum responses around the average characteristic frequencies of the neurons contained within each voxel. The resulting recentered, array-average BOLD response tuning curve (Fig. $6 \mathrm{~F}$, right panel) was approximately Gaussian-shaped with a width of $17.8 \mathrm{ERBs} F W H M$, similar to the response tuning widths observed in the anterior gradient ROI in the experimental data (parameter $\delta$ was fine-tuned to 16 ERBs to achieve this similarity). When we assumed that the only source of adaptation was neuronal $(\kappa=0.35$ and $\gamma=+\infty)$, the adapted BOLD responses to the probe again showed a localized trough around the probe frequency (Fig. 6G, left 
panel), and the array-average adaptation tuning curve showed a corresponding peak at the probe frequency (Fig. 6G, right panel), despite the unadapted probe response varying widely in amplitude across voxels. The width of the array-average adaptation tuning curve was again 7.2 ERBs, approximately $\sqrt{2}$ times the underlying neuronal tuning width, as was the case for the non-topographic model without BOLD non-linearity (compare with Fig. 6C, right panel). If, on the other hand, we assumed that the only source of adaptation was due to BOLD non-linearity (by setting $\kappa$ to zero and $\gamma$ to 2.5), the resulting BOLD adaptation effect also became broadly tuned (Fig. 6H), rather than being frequency-independent, as was the case in the nontopographic model. This broad frequency tuning can be explained by a combination of two effects: on the one hand, the peak of the BOLD adaptation effect varied between voxels and corresponded to each voxel's preferred frequencies, rather than to the probe frequency (compare with Fig. 6F). This is because suppression due to BOLD non-linearity increases with a voxel's response to the adaptor and is therefore strongest when the adaptor frequency matches the voxel's preferred frequency. On the other hand, because the unadapted probe response varies between voxels, voxels contribute differently to the array-average BOLD adaptation tuning curve (Fig $5 \mathrm{H}$, right panel): voxels preferring frequencies close to $3839 \mathrm{~Hz}$ respond more strongly to the probe and thus show more adaptation in absolute terms. The combination of these two effects led to the array-averaged BOLD adaptation tuning curve appearing tuned to the probe frequency, but with a much broader tuning width than for the model with purely neuronal adaptation (22.2 ERBs FWHM). With the specific parameters chosen for this simulation (again, set to approximately reproduce the experimental results), this apparent BOLD adaptation tuning was slightly larger than the adaptation tuning width actually observed in primary auditory cortex (when fitting the adaptation tuning with a simple Gaussian, compare with Fig. $4 \mathrm{C}$ ). Finally, when assuming both neuronal adaptation and BOLD non-linearity as sources of BOLD adaptation, the voxelwise and array-average adaptation tuning curves (Fig. 61) showed a superposition of the broader and more narrowly tuned adaptation effects seen in the previous two models (Fig. 6l, right panel). Fitting the arrayaveraged adaptation tuning curve with a simple Gaussian (Fig. 61, right panel) yielded an intermediate adaptation tuning width of 14.0 ERBs, which, as for the measured data, was similar to the width of the array-average response tuning curve (17.8 ERBs; compare with Fig. $6 F)$. Fitting the BOLD adaptation tuning curve with a Gaussian with offset yielded a narrower tuning width (10.3 ERBs), again similar to the tuning width obtained in primary auditory cortex (compared with Fig 4C, dashed line), but wider than the tuning width of the purely neuronal BOLD adaptation effect (7.1 ERBs) and corresponding underlying neuronal tuning (5 ERBs).

The above informal simulations suggest that fMRI adaptation tuning systematically overestimates the underlying neuronal adaptation tuning, and that the amount of overestimation depends on the topographical organization of the cortical area under study 
land therefore on whether frequency-specific and frequency-independent can be disentangled using a simple Gaussian model with offset). In Figure 7, we explore these biases more systematically by looking at how BOLD response and fMRI adaptation tuning width estimates (obtained by fitting Gaussian models) compare with the true underlying neuronal tuning for a more comprehensive range of model parameters.
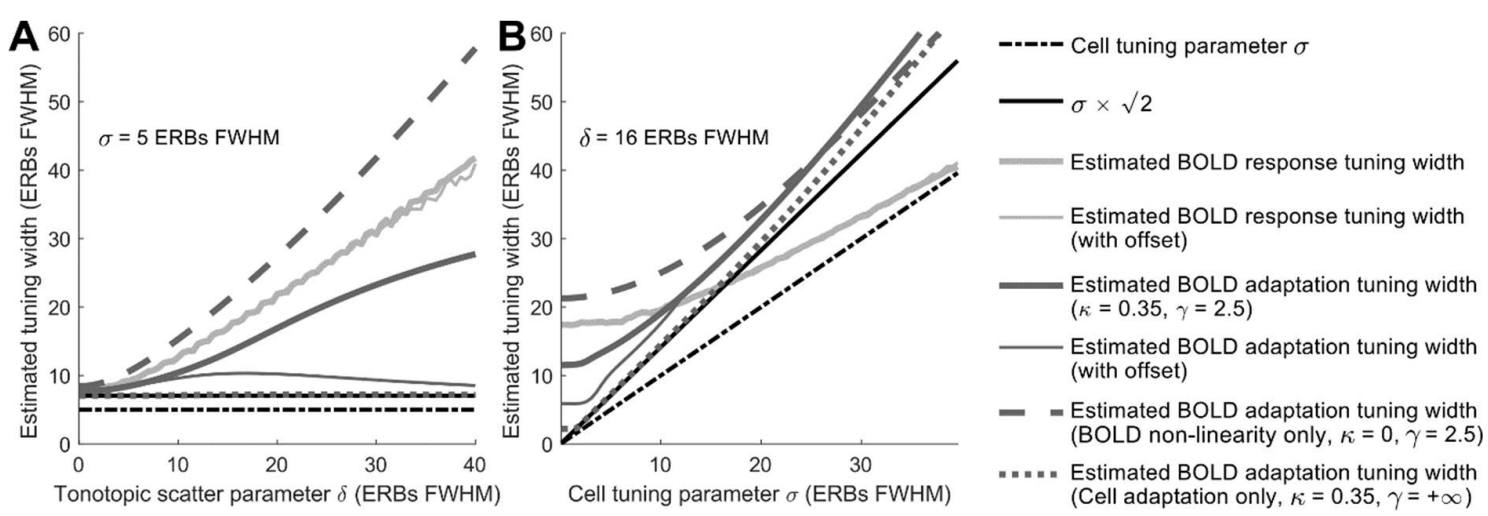

Figure 7: Estimated model BOLD response and adaptation tuning width for different values of the model's free parameters. A. Estimated array-average BOLD response tuning width (light gray traces) and BOLD adaptation tuning width (dark gray traces), plotted as a function of the tonotopic scatter parameter $\delta$, for three model versions: a model only implementing neuronal adaptation ( $\kappa=0.35, \gamma=+\infty$; dotted light gray trace), a model only implementing BOLD non-linearity ( $\kappa=0, \gamma=2.5$; dashed light gray trace) and a model implementing both ( $\kappa$ $=0.35, \gamma=2.5$; solid light gray trace). All three models had a neuronal tuning width $\sigma=5$ ERBs FWHM (represented as the thin dashed-dotted black trace) and produced identical BOLD response tuning width estimates (since these only depend on $\sigma$ and $\delta$ ). Response and adaptation tuning widths were obtained by fitting either a simple Gaussian (thick gray traces) or a Gaussian with offset (thin gray traces). For models implementing either only neuronal adaptation or only BOLD non-linearity, only the simple Gaussian tuning width is shown because estimates were identical for both methods. The thin solid back trace represents the neuronal tuning width multiplied by $\sqrt{2}$. B. Same as A, but keeping $\delta$ constant at 16 ERBs and varying $\sigma$ between 0.1 and 40 ERBs.

Figure 7A shows BOLD response and fMRI adaptation tuning width estimates for a continuum of models with decreasing topographic organization (increasing tonotopic scatter parameter $\delta$ ), when the neuronal tuning width is relatively narrow ( $\sigma=5$ ERBs FWHM, horizontal dashdotted black trace). As expected, the BOLD response tuning width consistently overestimates the actual neuronal tuning width, whether it is estimated using a simple Gaussian (thick lightgray trace) or a Gaussian with offset (thin light-gray trace), and the amount of bias increases systematically with increasing tonotopic scatter. The minimal amount of bias, observed when there is no tonotopic scatter $(\delta=0)$, is related to the magnitude of the tonotopic gradient within each voxel: the steeper tonotopic gradient, the greater the range of neuronal characteristic frequencies within a given voxel, and thus the larger the amount of overestimation (data not shown). The estimated fMRI adaptation tuning width (thick and thin solid dark-gray traces) also consistently overestimates neuronal tuning width, and the size of the bias can be explained by the two effects already illustrated in Fig. 6l. Even in the absence of BOLD non-linearity, i.e. if adaptation was purely neuronal ( $\kappa=0.35$ and $\gamma=+\infty$, as in Fig. $6 \mathrm{G}$ ), 
the adaptation tuning width obtained using a simple Gaussian (horizontal dotted dark-gray trace in Fig. 7A) would overestimate the underlying neuronal tuning width by a constant factor of $\sqrt{2}$, irrespective of the amount of tonotopic scatter, $\delta$ (compare with the thin solid back horizontal trace, representing $\sqrt{2} \times \sigma=7.07$ ERBs). Conversely, if adaptation was purely due to BOLD non-linearity $(\kappa=0$ and $\gamma=2.5$, as in Fig. $6 \mathrm{H}$ ), the estimated adaptation tuning width (dark-gray dashed trace) would increase strongly with increasing tonotopic scatter, similar to the BOLD response tuning width (compare with light-gray traces). As a result, for models using a combination of neuronal adaptation and BOLD non-linearity (as in Fig. 61), estimated adaptation tuning width is intermediate between the previous two scenarios when estimated using a simple Gaussian model (thick solid dark-gray trace), and overestimation of the underlying neuronal tuning width increases overall with decreasing topographic organization. Attempting to measure the tuning width of only the frequency-specific adaptation component by fitting a Gaussian with offset (thin solid dark-gray trace) only partially reduces the bias, although this strategy becomes more successful for large values of the tonotopic scatter parameter (i.e. for non-topographically-organized cortical regions).Figure 7B shows that similar biases are obtained for a wide range of actual neuronal tuning widths, $\sigma$, when the tonotopic scatter $\delta$ is fixed atl 6 ERBs (and $\kappa$ and $\gamma$ are set to the same values as in Fig. 7A). Although the estimated BOLD response tuning width converges towards the true cell tuning width for larger values of $\sigma$ (i.e. the contribution of the tonotopic scatter becomes negligible for widely tuned neurons), adaptation tuning width overestimates the underlying neuronal tuning width at all tested values of $\sigma$. For purely neuronal adaptation, the discrepancy between actual neuronal tuning width and estimated adaptation tuning width again corresponds to a multiplicative constant of $\sqrt{2}$ (or slightly greater for $\sigma>20$ ERBs). When introducing BOLD non-linearity, the discrepancy increases substantially, particularly for $\sigma$ values below about 20 ERBs.

Figure 7 illustrates how, in our model, the apparent tuning of adaptation tuning effect due to BOLD non-linearity depends on the range of characteristic frequencies of the cells contained in each voxel. This range depends both on the tonotopic scatter and on the magnitude of the tonotopic gradient. While tonotopic scatter cannot be directly measured using fMRI, we measured the average tonotopic gradient in each of our four ROls to see if this parameter could, by itself, explain the differences in adaptation tuning observed across the ROls. Average tonotopic gradient magnitudes were $2.34 \pm 0.24,2.08 \pm 0.13,2.26 \pm 0.24$ and $2.10 \pm 0.21 \mathrm{ERB} / \mathrm{mm}$ from the most anterior to the most posterior ROI, respectively. They did not significantly differ between ROls and their pattern did not match the observed pattern of BOLD adaptation tuning width shown in Fig. 4C (irrespective of whether or not the Gaussian fit included a constant offset). 


\section{Can we infer average single-neuron frequency selectivity in human auditory cortex from fMRI adaptation data?}

Our model simulations suggest that, for tonotopically-organized neuronal populations, the adaptation effect produced by BOLD non-linearity can appear frequency-specific. This apparent tuning in BOLD adaptation can be difficult to distinguish from that due to genuine neuronal adaptation, leading to overestimating adaptation-inferred neuronal tuning. To disentangle neuronal and BOLD contributions to adaptation tuning, and attempt to infer underlying average single-neuron tuning properties, we fitted our model of fMRI adaptation to the observed group- and ROI-average response and adaptation tuning curves. Three of the model's four free parameters - the tonotopic scatter, $\delta$, the neuronal tuning, $\sigma$, and neuronal adaption strength, $\kappa$ - were assumed to vary across ROls, whereas the strength of BOLD nonlinearity, $\gamma$, was assumed to be constant across ROls. We fitted the model 1000 times with random initial free parameters taken from an extensive range.

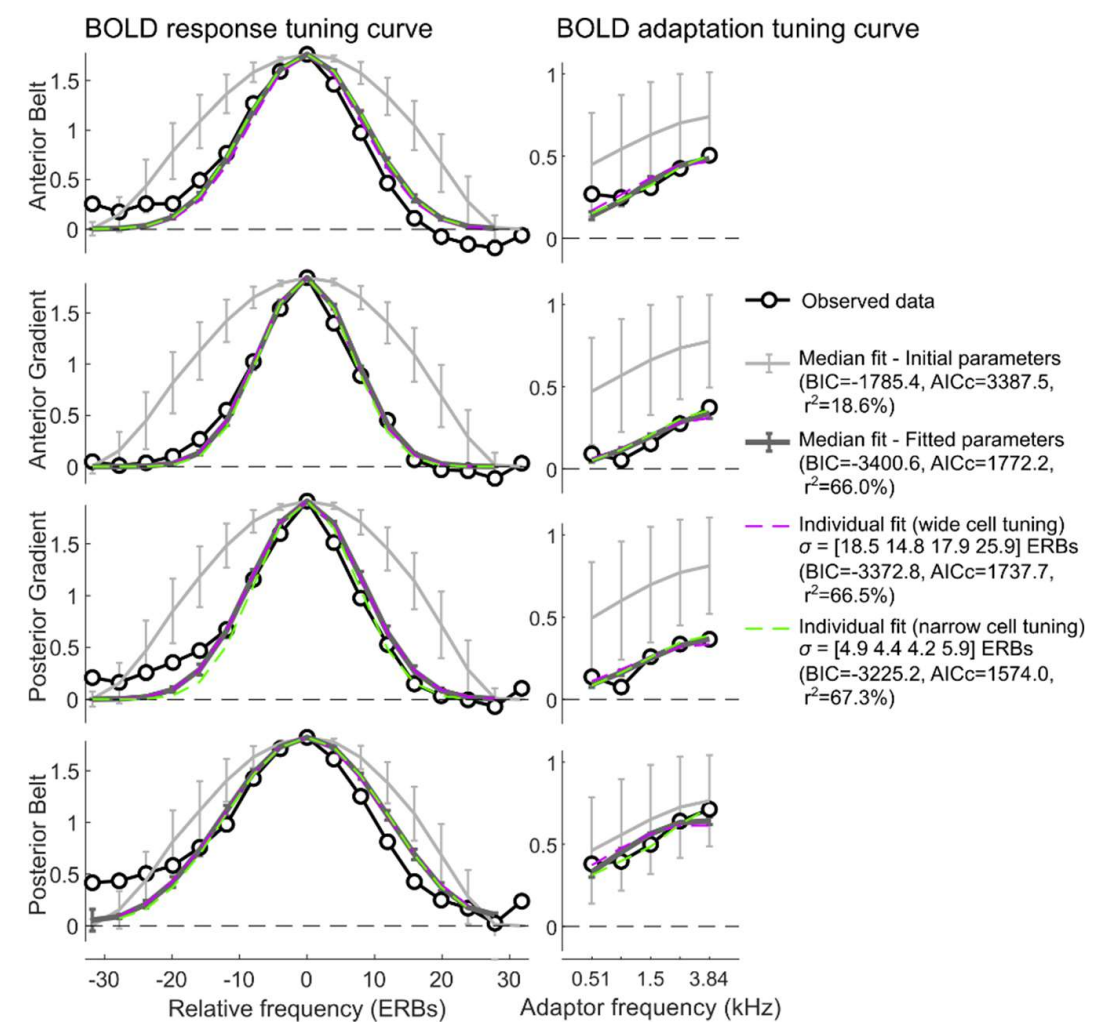

Figure 8: Modeled BOLD response and adaption tuning curves superimposed on experimental ROI-average tuning curves. Black traces with open circles are actual tuning curves, replotted from Fig. 4B-C. Thick gray traces are the median of 1000 best-fitting model tuning curves and thin light gray traces are the median of the correspond 1000 initial parameter tuning curves. Error bars are interquartile intervals across the 1000 fits. Purple and green dashed traces correspond to two example individual fits, with parameters highlighted in Fig. 9. The purple trace corresponds to an individual fit with wide neuronal tuning parameters and the green trace to a fit with narrow neuronal tuning parameters. 
Figure 8 shows the median modelled response and adaptation tuning curves obtained from these 1000 fits and from the corresponding initial parameters. The median fitted curves (thick dark gray traces) fitted the data better than tuning curves modelled using random initial parameters (thin light gray traces), average $r^{2}=66.0$ vs $18.6 \%$, but did not capture all the features of the experimental data. In particular, it did not reproduce the asymmetrical offset of the response tuning curves observed outside the anterior gradient ROI (as expected, since tuning is entirely symmetric in the model), nor the frequency-independent adaptation offsets of the adaptation tuning curves (contrary to expectation).
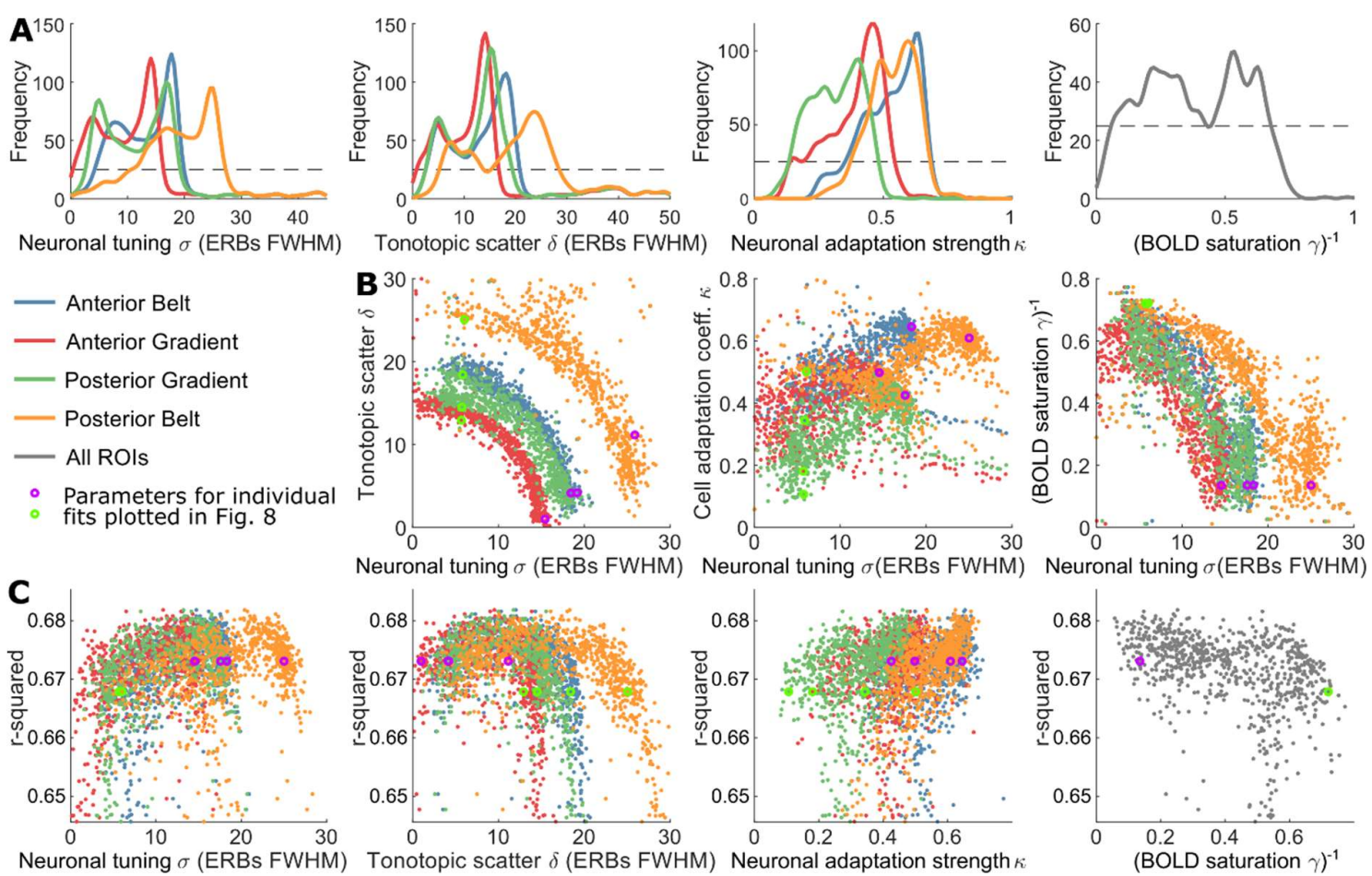

Figure 9: Distributions and goodness-of-fit of best-fitting parameters across 1000 fits with random initial parameters. A. Distributions of the best-fitting parameters for the 4 free parameters in the 4 core and belt ROls ( 10 free parameters since $\gamma$ was common to all ROIs). Distributions were smoothed using kernel density estimation. The thin dashed black line represents the uniform distribution of random initial parameters. B. Selected joint distributions of pairs of free parameters across the 1000 fits, plotted as scatter plots for each of the 4 ROIs. C. Scatter plots of the 4 free parameters and $r^{2}$ values across the 1000 fits for each of the 4 ROIs. In B and C, parameters sets corresponding to the two individual fits plotted in Fig. 8 are highlighted in purple and green, respectively.

Figure 9A shows the distributions of all fitted parameters across the 1000 fits, compared to the (uniform) distribution of random initial parameters (dashed line). All fitted parameter distributions were narrower than the corresponding initial parameter distributions, but they were typically wide and bimodal, and showed marked differences between ROls. The anterior gradient ROI had the lowest values for the neuronal tuning parameter $\sigma$ and the tonotopic scatter parameter $\delta$ (both ranging between 1 and 16 ERBs FWHM) and the highest values for the neuronal adaptation strength $\kappa$ (ranging between 0.35 and 0.7 , at par with the posterior 
gradient ROI). The posterior belt ROI had the corresponding highest and lowest values for these three parameters. The reciprocal of the BOLD saturation parameter $1 / \gamma$, common to all ROls, ranged between 0.05 and 0.7 (corresponding to $\gamma$ parameter values between 2.5 and $136 \%$ signal change when rescaled to the measured BOLD tuning curves). Scatter plots across the 1000 fits for pairs of fitted parameters (Fig. 9B) indicated several trade-offs in fitting these free parameters, which partly explain the wide and bimodal distributions obtained for individual fitted parameters. The neuronal tuning parameter $\sigma$ and the tonotopic scatter parameter $\delta$ (leftmost panel) were negatively related with what looked like a quadratic relationship $\left(\sigma^{2}+\right.$ $\delta^{2}=$ constant). On the other hand, $\sigma$ was positively related to both the neuronal adaptation strength $\kappa$ and the BOLD saturation parameter $\gamma$. These trade-offs in fitted parameters suggest a continuum of model solutions between two extremes: a model with narrow neuronal tuning (< 10 ERBs in all ROIs), high tonotopic scatter (14 to 26 ERBs, depending on the ROI), low neuronal adaptation strength (20 to 50\%) and low BOLD saturation (i.e. more non-linearity, $1 / \gamma$ between 0.4 and 0.66 , corresponding to saturation parameter between 2.7 to $4.5 \%$ signal change), and an opposite model with relatively wide neuronal tuning, low tonotopic scatter, high neuronal adaptation strength and less BOLD non-linearity $(\sigma=14$ to 26 ERBs, $\delta<10$ ERBs, $\kappa=030$ to $70 \%, 1 / \gamma<0.3$ or $\gamma>6 \%$ signal change). Two example model fits (one at each extreme of this continuum) are illustrated in Figure 8 (green and purple dashed traces, with circles of corresponding colors in Fig. 9B showing the respective parameters). Both fits were very similar and closely followed the median fitted model, with the largest (minor) difference occurring for the response tuning curve in the posterior gradient ROI and for the adaptation tuning curve in the posterior belt ROI. The model with broad neuronal tuning (purple trace) followed the response tuning curve slightly more closely, while the model with narrow neuronal tuning (green trace) followed the adaptation tuning curve more closely, following the frequency-independent minimum adaptation offset at low adaptor frequency values. The two example models were otherwise very similar in other ROIs and neither captured the minimum adaptation offset in any of the other three ROIs. The reason none of the model versions could capture the apparent frequency-independent BOLD adaptation offset is that the model has to explain both the adaptation and response tuning curves, but that the limited amount of scatter (<30 ERBs) required for a tuned (tonotopically-organized) BOLD response is incompatible with a frequency-independent BOLD adaptation component (compare with Fig. 6B-D), at least in our implementation. Overall, models with wide neuronal tuning, low tonotopic scatter and high neuronal adaptation strength tended to have slightly better goodness of fit (Fig. 9C).

\section{Discussion}

We used an fMRI adaptation paradigm to probe neuronal frequency tuning in human primary and secondary auditory cortex and demonstrated frequency-specific BOLD adaptation, such 
that the amount of adaptation increased as the frequency of the adaptor approached that of the probe. The shape and width of the obtained fMRI adaptation tuning curves, compared to those of the BOLD response tuning curves, suggested that fMRI adaptation selectivity does not directly reflect the underlying neuronal selectivity, as is often assumed. Computational modelling results suggest at least two mechanisms by which population adaptation tuning can appear wider than the underlying neuronal tuning. Directly fitting our model to the fMRI adaptation data yields insights into the plausible range of average neuronal frequency tuning in human core and belt auditory cortex.

\section{Biases in measuring population adaptation tuning curves}

Although we did observe frequency-selective adaptation in auditory cortex, as expected since most auditory cortical neurons are selective to frequency [e.g. 45], the tuning of this effect (as estimated by fitting a simple Gaussian) was larger than expected from previous unit recordings in both humans and other mammals (see next section). It was also at least as wide as the average voxelwise frequency tuning of the BOLD response, which would be expected to be wider than that of individual auditory neurons since each voxel includes a large population of neurons of varying characteristic frequency (see introduction). Our model simulations suggest at least two reasons why population (fMRI) adaptation frequency may overestimate neuronal tuning.

First, we found that population adaptation tuning overestimates the tuning of individual neurons by a factor $\sqrt{2}$ (see Fig. 6C,G and Fig. 7), even in the absence of any other confounding effect (such as BOLD non-linearity). In the supplementary discussion, we formally derive the value of this bias $(\sqrt{2})$, and we only give an intuitive explanation for why population adaptation tuning within a voxel should be expected to be wider than the adaptation tuning of single neurons here. The adaptive suppression effect observed in our fMRI adaptation paradigm is only contributed to by neurons that respond to the high-frequency probe (as can be seen in Fig. 3, where significant adaptation effects are mostly restricted to high-frequencypreferring regions). While the strongest contribution to this suppressive effect would be from neurons that prefer the probe's frequency, significant response would also come from neurons that prefer other frequencies, but still respond to the probe. For these neurons, the maximum adaptation effect would be observed at their characteristic frequency (because we assumed that a neuron's frequency tuning reflects its response tuning) instead of the probe frequency, widening the observed population adaptation tuning curve. In other words, the measured population adaptation tuning curve does not correspond to the average tuning curve of neurons preferring the probe frequency, but to the average tuning curve of neurons with a range of frequency characteristics around the probe frequency, weighted by their contribution of the adaptation effect (which itself depends on the average tuning width of the adaptation effect), widening the tuning of the population adaptation effect compared 
of the tuning of each single neuron. This bias is expected irrespective of the spatial or topographic distribution of characteristic frequencies, as demonstrated by our simulations (compare Fig. 6C and 6G). Our results therefore suggest that previous studies that have attempted to quantitatively measure neuronal selectivity for stimulus features from macroscopic measurements [e.g. 14, 15, 46] might have overestimated its tuning width. The existence of this bias does not necessarily call into question fMRI studies that have used adaptation paradigms to qualitatively demonstrate the existence of neuronal selectivity, rather than attempting to measure neuronal tuning curves [e.g. 47, 48].

The second bias suggested by our model simulations is due to the contribution of BOLD nonlinearity to $f M R I$ adaptation effects. It is well known that the BOLD response is not linear and that the BOLD response to two stimuli presented in close succession is subadditive [e.g. 35]. Although part of BOLD non-linearities might be neuronal in origin $[49,50]$, they probably also include non-neuronal (e.g. vascular) contributions [51], which should not be expected to show stimulus (frequency) specificity. We modelled this non-linearity by applying a saturation function to the neuronal response and found that its effect on the population adaptation tuning could be mistaken for the effect of frequency-specific adaptation. When a pair of stimuli (e.g. an adaptor-probe pair) is presented, the amount of suppression due to this nonlinearity should depend on the relative BOLD response to each of the stimuli in the pair: more suppression is expected if a region responds strongly to both stimuli in the pair (e.g. when the adaptor and probe frequencies match), than if the response to at least one of the stimuli is weak. In a cortical region in which stimulus specificity is not topographically organized, any part of the region would respond equally to all stimuli along the stimulus feature and so the same amount of suppression should be observed irrespective of the identity of the stimuli in the pair. In this case, the adaptive effect of BOLD non-linearity would appear fully stimulusindependent at the population level and although this could lead to overestimating adaptation tuning width, this effect could also easily be separated from genuine neuronal adaptation effects, which are tuned to the stimulus feature. This is presumably the case for features such as stimulus orientation in primary visual cortex, which is not (or only weakly) topographically distributed at the spatial resolution used here or at conventional fMRI resolution. When the stimulus feature of interest is topographically organized however (or presents important spatial biases in feature representations), like frequency in auditory cortex, our model suggests that the adaptation effect due to non-neuronal non-linearities would nevertheless appear tuned to the probe stimulus, mimicking the expected frequency-specific effect of neuronal adaptation. This is because in a topographically-organized region, different locations respond differentially to different stimuli along the feature dimension, and so suppression due to BOLD non-linearity is stronger when the adaptor matches the probe than when it doesn't, appearing tuned to the probe stimulus in regions that contribute most to the 
observed adaptation tuning curve (those responding to the probe frequency). Unlike tuning due to genuine neuronal adaptation, this apparent tuning of the BOLD non-linearity does not depend only on the tuning of individual cells, but rather on the tuning of the BOLD response itself, which also reflects the spatial distribution of stimulus preference (i.e. the spatial gradient and scatter of the stimulus feature of interest), as well the spatial resolution of the macroscopic measurement (how many cells contribute to the signal in a given voxel). Therefore, this spurious adaptation tuning is expected to be wider than the tuning due to purely neuronal adaptation.

Beyond these two biases, our fMRI adaptation data also suggest the possible existence of a frequency-independent component in the BOLD adaptation tuning curve. This frequencyindependent adaptation component cannot be explained by the above non-neuronal nonlinearity in our model, because adaptation due to BOLD non-linearity is expected to be tuned in tonotopically-organized cortex. What mechanism could explain this frequencyindependent adaptation? One possibility is that the neuronal response tuning curves themselves include a frequency independent component that would then be reflected in the adaptation tuning curve. We in fact do observe such frequency-independent response in the $B O L D$ response tuning curves and their amplitudes in different ROls does seem to match the amplitude of the adaptation offset in most ROls (compare the left offsets of the response and adaptation tuning curves in Fig. 8). Another possibility is that there is an active suppression of $B O L D$ responses by frequencies not included in the response tuning curve, maybe reflecting lateral inhibition mechanisms, as observed in visual cortex [52]. Including either of these two mechanisms in our model may result in a decrease in the estimated neuronal tuning width, since they would account for the enlargement of the adaptation tuning curves due to this frequency-independent component. Note however that we tested a limited number of adaptor frequencies far from the probe frequencies and that model comparison indices (weakly) favored simple Gaussian models of the fMRI adaptation tuning curves (without frequency-independent component). Further experiments with adaptor frequencies further away from the probe (at both lower and higher frequencies) will be necessary to ascertain the existence of this frequency-independent adaptation component.

\section{Frequency tuning width estimates in core and belt auditory cortex}

To account for the biases highlighted in the previous section and estimate average neuronal tuning width in different regions of auditory cortex, we fitted our adaptation model to the fMRI adaptation data. While the presence of trade-offs between the free parameters of the model prevented us from obtaining a satisfactorily narrow range of parameter values for the average neuronal tuning width, we could still estimate a range of plausible values for each auditory area. The obtained values are consistent with neuronal tuning being narrower in core 1-16 ERBs in the most selective region in the anterior gradient ROI overlapping most with core auditory cortex on Heschl's gyrus) than belt auditory cortex (10-27 ERBs for the least tuned 
region in the posterior belt $\mathrm{ROI}$ on planum temporale), as has been shown in non-human primates $[53,54]$.

How do these values compare with electrophysiological estimates of tuning width in humans and other primates? One study recording from single neurons in the primary auditory cortex of pre-surgical epileptic patients [2] reported that the majority of neurons were more finely tuned than auditory nerves (i.e. < 1 ERB), which is below the lower bound that we find in the present study. Electrophysiological studies in other primates however have reported wider tuning widths. We digitized cortical tuning widths from seven studies that reported single- or multi-unit tuning width measurements in either core or belt areas in non-human primates (see Methods section for details). Based on these data, we estimate that the median population tuning width across studies was between 2.8 and 4.9 ERBs in core auditory cortex (depending on the precise assumption made when converting these tuning width to a format compatible with our measurement, see supplementary methods) and between 3.5 and 6.0 ERBs in belt auditory cortex (values varied widely across individual studies, possibly due to differences in species and anesthetics, see supplementary Table 1). While these values are compatible with those we derive from fitting our adaptation model for core auditory cortex (between 1 and 16 ERBS) and the anterior part of the human belt region (between 4 and 20 ERBs), they are narrower than the lower bound of the range we estimate for the posterior belt region (10 to 27 ERBs). Furthermore, it has been suggested that many non-human primate neuronal tuning width estimates have been overestimated because they were obtained under anesthesia [1, but see 55] and are in fact no wider in awake preparations than those reported from human auditory cortex by Bitterman, Mukamel [2]. If this is correct, our estimates may be wider than those obtained from direct recordings in auditory cortical cells in both human and non-human primates.

We also compared our estimates to frequency tuning width estimates digitized from three human EEG studies using an adaptation paradigm comparable to ours (see supplementary Fig. 3). These estimates ranged between 15.4 and 20.8 ERBs, with a mean of 18.0 ERBs. Taking into account the $\sqrt{2}$ bias described in the previous section, this would correspond to an average neuronal tuning width of $12.8 \mathrm{ERBs}$. Since these adaptation data were obtained from $\mathrm{N} 1$ and/or P2 ERP components, which are generated both in primary and secondary auditory cortex [23], this is compatible with the range of tuning widths we obtained using fMRI adaptation in both the anterior and posterior belt regions. Therefore, it seems that neuronal frequency selectivity estimates obtained using an adaptation paradigm are wider than those obtained by direct neuronal recordings in humans or non-human primates, even when accounting for potential biases due to the macroscopic nature of EEG and fMRI signals. This discrepancy may be due to the fact that both BOLD and EEG signals are more sensitive to peri-synaptic activity than to spike rates used in unit recording $[56,57]$. Intracellular recordings 
in the auditory system show that there is considerable subthreshold synaptic activity in response to frequencies beyond auditory neurons' classical frequency receptive field [e.g. 58]. This subthreshold synaptic activity may contribute to neuronal adaptation and widen neuronal frequency adaptation tuning curves (see next section for a more in-depth discussion).

Given the wide range of possible tuning widths obtained by fitting our model, comparisons with direct recordings remains highly speculative at this point. This uncertainty in the value of the fitted frequency tuning parameter was clearly linked to the uncertainty in the value of the other free parameters of the model, in particular, the tonotopic scatter and the relative strength of neuronal and non-neuronal adaptation: finer tuning estimates are obtained when assuming more tonotopic scatter (more variability in the characteristic frequencies at a given location) and a stronger contribution of non-neuronal non-linearity. Therefore it might be possible to narrow the range of tuning width estimates by incorporating information about these parameters, for instance by using more realistic models of neurovascular coupling the BOLD non-linearity [e.g. 59] and quantitative estimates of tonotopic scatter and neuronal adaptation strength. For instance, two-photon calcium imaging studies have shown a lack of tonotopic organization on a fine scale in mammalian auditory cortex [60, 61], suggesting a relatively large value for tonotopic scatter, and thus a narrower tuning (according to our model).

\section{Assumptions and caveats}

An important assumption of our adaptation model is that the amount of neuronal adaptation following a stimulus is proportional to the response of the neuron to this stimulus. In other words, we assume that response and adaptation tuning curves have the same shape at the level of a neuron. This would be the case if adaptation was mediated by spiking fatigue [a decrease in spike rate proportional to the spike rate in response to the adaptor, due for instance to hyperpolarization, 62]. Electrophysiological studies in the primate visual system (mostly the inferior temporal cortex specialized in object recognition) have shown that the adaption tuning does not necessarily match neuronal tuning [63-65], and therefore that adaptation effects must also be underpinned by synaptic mechanisms, such as synaptic depression [66]. Furthermore, modelling work suggests that adaptation effects that are purely proportional to the response to stimuli cannot alone account for $\mathrm{fMRI}$ adaptation effects observed in some parts of visual cortex [67]. Similarly, in mammalian auditory cortex, the tuning of frequency adaptation (forward masking), although centered on the characteristic frequency of neurons, has been shown to differ from response tuning [e.g. 68]. Moreover, when using probe frequencies that differ from the characteristic frequency of neurons, adaptation tuning curves has been shown to be centered on the probe frequency rather that the characteristic frequency [69], suggesting that adaptive synaptic mechanisms such as synaptic depression might dominate cortical frequency adaptation [e.g. 70, 71]. 
Although there is overwhelming evidence that spiking fatigue cannot by itself account for (frequency) adaptation in cortex at the neuronal level, this does not necessarily invalidate our conclusions, but it would strongly impact how our adaptation model and its parameters should be interpreted neurophysiologically. In spiking fatigue, suppression is proportional to the output response of a neuron. By analogy, in synaptic depression, suppression would presumably be proportional to cellular activity at a given synapse. Therefore, adaptation tuning would directly reflect the tuning of cellular activity at this synapse. If cortical frequency adaptation is dominated by (suppressive) synaptic mechanisms, we could still keep the assumption that suppression is proportional to response if we assume that the frequency-tuned units in the model correspond to synapses rather than neurons, and responses correspond to synaptic activity rather than output neuronal activity (spiking). The latter notion would in fact be more consistent with the predominantly peri-synaptic origin of the BOLD signal $[56,57]$. In this new interpretation, the tuning width parameter of our adaptation model would measure the tuning width of frequency-selective synapses rather than that of the output (spike-rate) of frequencyselective neurons. In this case, the adaptation tuning measured at a given voxel using fMRI would correspond to the average output tuning of pre-synaptic neurons that project to this area. But given that cortical connections are predominantly local, input and output tuning might not differ very much at the level of a voxel [12]. The other parameters of the model, such as the tonotopic gradient and scatter would now quantify the spatial distribution of frequencytuned synapses (rather than neurons). While there is no reason to think that the tonotopic gradient for frequency-selective synapses and neurons should differ, the scatter parameter for synapses might be expected to exceed its neuronal equivalent, since the synaptic subthreshold tuning curves have been shown to be wider than spike-rate tuning curves [e.g. 58].

Note that it is currently unclear how much each of the two classes of adaptation mechanisms (spiking fatigue and suppressive synaptic mechanisms such as synaptic depression) contribute to frequency adaptation in cortex. The contributions of each mechanism may also depend on the delay between adaptor and probe, since the time course of the two mechanisms differ. Consistent with this idea, the relationship between adaptation and neuronal response tuning in auditory cortical cells changes with the delay between adaptor and probe [68], with adaptation tuning most similar to response tuning at short delays. However, the exact contribution of SOA (200-300 ms in our case) and ISI (30 ms in our case) to this effect are currently unclear.

Other mechanisms than response fatigue and suppressive synaptic mechanisms may be involved in adaptation, including those leading to facilitation effects [70, 72] and more complex mechanisms involving a more extensive network of connections between neurons [e.g. 73, 74]. While our model does not allow for facilitation effects of the adaptor onto the 
probe response, the fact that we observe suppression adaptation at the population level suggests that the latter dominate.

An related interpretational issue, common to all adaptation studies, is that of inherited adaptation [12, 24, 75]: does the observed adaptation selectivity reflect the neuronal selectivity within the recorded region, or does the adaptation effect already exist in upstream regions and is transmitted as is to the recorded region? In the auditory system, adaptation effects are known to be more short-lived in subcortical than cortical neurons and so adaptation effects observed in cortex have often been thought to be mainly cortical in origin [71]. However, as we used a relatively short ISI (30 ms) between adaptor and probe (but relatively long SOAs, 200-300 ms), we cannot exclude that at least some of the observed adaptation effect is inherited from subcortical nuclei. Further experiments that parametrically vary both ISI and SOA between adaptors and probes would clarify this question. Longer delays could be used to ensure that the observed adaptation effects exclusively reflect cortical tuning. In any case, the fact that we observed differences in adaption tuning between different cortical regions suggest that the observed adaptation cannot be entirely inherited from subcortical adaptation.

\section{Conclusion}

Our results suggest that fMRI adaptation is suitable for measuring neuronal frequency tuning properties in human auditory cortex, provided population effects and the non-linearity of BOLD response are taken into account.

\section{Material and methods}

This study involved 11 normal-hearing participants with no history of neurological or otological disease ( 3 males; age range: $29.9 \pm 7.4$ ). All participants gave prior-informed written consent. The procedures complied with the Declaration of Helsinki guidelines (Version 6, 2008) and were approved by the Ethics Committee of the University of Nottingham's School of Medicine. Some of the current data (for conditions not involving adaptation) have been presented previously [4], with the current participants 1 to 11 corresponding to participants 2 to 12 in the previous study (previous participant 1 did not complete all adaptation conditions).

All scanning was performed on a Philips Achieva 7T system with a birdcage transmit, and 32channel receive coil (Nova Medical, Wilmington, MA). Participants watched a self-chosen silent subtitled movie to stay alert and were told that they could ignore all sound stimuli.

\section{Functional MRI acquisition, acoustic stimuli and pre-processing} $B O L D$ fMRI data were acquired using a sparse (TR $=7.5 \mathrm{~s}$ ) gradient-echo EPI sequence (TE $=25$ $\mathrm{ms}, \mathrm{SENSE}=3, \mathrm{FA}=90^{\circ}$ ) with $1.5 \mathrm{~mm}$ isotropic resolution. The acquisition stack consisted of 20 contiguous axial slices oriented parallel to the Sylvian fissure. The experimental stimuli were 
presented in the silent periods separating successive fMRI acquisitions, starting $1.28 \mathrm{~s}$ after the end of the preceding acquisition and continuing for $5 \mathrm{~s}$, until the start of the next acquisition (Fig. 1).

Each 5-s trial contained either no experimental stimuli ("silent" trials), or a train of 10 identical stimuli, presented once every $500 \mathrm{~ms}$ (Fig. 1). Each stimulus consisted either of an adaptorprobe pair (AP trials), or the adaptor or probe presented in isolation (A-only or P-only trials). Both the adaptors and probe were narrowband noises with a bandwidth equal to the normal auditory filter bandwidth at the center frequency. The auditory filter bandwidths are based on behavioral notched-noise measurements [25] expressed as equivalent rectangular bandwidths, or ERBs (i.e., the widths of rectangular filters with the same peak gain and total power), and approximated as a linear function, $\operatorname{erb}(f)=24.7 \cdot(4.37 \cdot f+1)$, of the filter center frequencies, $f$. The frequencies of both the adaptors and probes were fixed within each trial. Across trials, the frequency of the probe was fixed at $3.839 \mathrm{kHz}$, whilst the frequency of the adaptor was varied across seven possible values $(0.251,0.505,0.899,1.501,2.423,3.839$ and $6.009 \mathrm{kHz}$ ), spaced evenly on a quasi-logarithmic frequency scale referred to as the ERBnumber scale [see 26, and https://en.wikipedia.org/wiki/Equivalent_rectangular_bandwidth], which approximates the human cochlear frequency scale by measuring the number of ERBs below each frequency [27]. For the AP trials, the adaptor frequencies were limited to the central five of these values $(0.505,0.899,1.501,2.423,3.839 \mathrm{kHz})$, yielding a total of 14 different trial types: silent, P-only, five AP trials and seven A-only trials.

Across different participants, the adaptors were either single 200-ms bursts (Fig. 1B; first five participants), or short sequences of four 50 -ms bursts separated by 30 -ms silent gaps (Fig. 1C; last six participants). The probe was a single 50-ms burst for all participants. In the AP trials, each probe followed the end of the respective preceding adaptor after a 30-ms silent gap. In the A-only and P-only trials, the adaptor and probe stimuli were presented in the same temporal positions relative to the offset of the preceding fMRI acquisition as in the AP trials. All stimulus bursts were gated on and off with 10-ms quarter-cosine ramps. The stimuli were generated digitally, converted to analogue voltage and presented through MR-compatible insert earphones (Sensimetrics S14) at a root-mean-square level of $70 \mathrm{~dB}$ SPL (sound pressure level). They were also equalized to compensate for the frequency transfer characteristics of the earphones, and presented in a continuous background of uniformly exciting noise [filtered to contain equal energy within all cochlear filters; 28 ] to roughly equate their sensation levels (SLS) across frequencies and participants. The noise was presented at $35 \mathrm{~dB}$ SPL per ERB, creating a constant hearing threshold of approximately $29 \mathrm{~dB}$ SPL [29].

Data were acquired in 8-min runs consisting of a total of 64 trials each, including four repetitions of each of the 13 non-silent (AP, A and P) trials and 12 repetitions of the silent trials. The SL- 
equalizing background noise was presented continuously throughout the run duration. The trials were presented in a pseudo-random sequence consisting of four successive random permutations of one of each of the non-silent trials and three silent trials, respectively. Most participants completed a total of six runs, except for participants 2 and 5 who completed eight and four runs, respectively.

The functional images were corrected for distortions due to dynamic $B_{0}$ inhomogeneities [see 4 for details], motion-corrected, high-pass filtered (cut-off $0.01 \mathrm{~Hz}$ ), converted to percent signal change and finally concatenated across runs. BOLD responses to each of the 13 sound conditions (seven A, five AP and one P condition) were estimated voxelwise by fitting a general linear model to the concatenated functional time series for each subject [see 4 for details].

\section{Structural MRI acquisition, functional-to-structural alignment and group normalization}

A whole-head, high-resolution T1-weighted structural volume was acquired using a phasesensitive inversion recovery (PSIR) sequence ( $T R=15 \mathrm{~ms}, \mathrm{TE}=6.1 \mathrm{~ms}, \mathrm{SI}=5000 \mathrm{~ms}$ ) with $0.6 \mathrm{~mm}$ isotropic resolution. This was used both to create a flattened representation of the supratemporal cortical surface around auditory cortex in each subject and hemisphere, and to estimate local intracortical myelin content. An unbiased, semi-quantitative measure of longitudinal relaxation rate (R1), which is indicative of cortical myelination, was obtained by dividing the sign-corrected T1-weighted PSIR volume voxelwise by the corresponding protondensity-weighted volume [see 4]. The resulting Rl volume was segmented to reconstruct individual three-dimensional cortical surfaces at 11 cortical depths [using Freesurfer v5.3, https://surfer.nmr.mgh.harvard.edu/, 30], which, in turn, were used to create the flattened cortical patches (using vistaSoft's mrVista, http://web.stanford.edu/group/vista/cgibin/wiki/index.php/MrVista). To project the functional data onto the cortical patches, the functional volumes were non-linearly registered with the T1-weighed PSIR volume [Using an additional $\mathrm{T}^{*}$-weighted FLASH volume with the same slice prescription as the functional volumes in an intermediate registration step, see 4 for details].

Functional parameter estimates for each of the 13 different sound conditions were also normalized to the MNI305 brain template (fsaverage) using spherical normalization of the 11 cortical surfaces in Freesurfer [31] and mrTools [32]. For all group analyses performed on spherically-normalized data, the left and the right hemispheres of each subject were treated as independent and data were aggregated across the left and the right hemispheres by projecting individual right hemisphere data onto the template's left hemisphere [see 4 for details]. 
In vivo parcellation of auditory cortex in individual hemispheres and definition of surface ROls

To delineate primary from non-primary auditory regions (commonly referred to as "core" and "belt") in individual hemispheres, we mapped local preferred frequencies, response tuning widths and cortical myelin content onto the individual flat cortical patches [4]. Local preferred frequencies and response tuning widths were derived from the voxelwise frequency response functions based on the BOLD responses to the seven different adaptor-only frequencies (henceforth referred to as "response tuning curves"). Preferred frequencies were obtained by calculating the tuning curve centroids, correcting them for the central bias caused by the finite range of adaptor frequencies, and then averaging them across the central five cortical depths. Response tuning widths were obtained by first recentering the voxelwise tuning curves on their respective centroid frequencies, averaging them across all cortical depths and smoothing them along the cortical surface (2D-Gaussian kernel with 3-mm FWHM, full width at half maximum). The voxelwise, recentred and smoothed tuning curves were then fitted with Gaussian-shaped functions (on the ERB-number scale). Given that the tuning curves were recentered, only the Gaussian scale (determining the size of the peak response) and spread parameters were fitted. Intracortical myelin content was estimated from the PSIR RI volumes by regressing out local cortical curvature and thickness and averaging across the middle five cortical depths. The preferred frequencies were used to define four contiguous surface regions of interest (ROIs) in each individual hemisphere. Reversals in local tonotopic gradient direction were identified using an unbiased automated procedure [33]. Each individual hemisphere showed a low-frequency reversal along the long axis of Heschl's gyrus (HG), flanked by two high-frequency reversals anteriorly and posteriorly [see Fig. 2A for an example hemisphere and 4 for all individual hemispheres]. We used these three reversals to define two ROIs corresponding to the two mirror-symmetric tonotopic gradients running across HG (henceforth referred to as "anterior and posterior gradient ROIs"). The anterior gradient ROI substantially overlapped regions of increased frequency selectivity (reduced response tuning width) and increased intracortical myelination observed along the long axis of $H G$, probably corresponding to the auditory primary, or core, region [see Fig. $2 B \& C$ and 4]. The posterior gradient ROI also overlapped the core region, but less so than the anterior gradient ROI. We then defined two additional ROIs posterior and anterior to the two gradient ROls, representing the secondary, or belt, auditory regions (henceforth referred to as "anterior and posterior belt ROIs"). These were defined as regions significantly responsive to any of the seven adaptor-only frequencies, but not belonging to either of the two gradient ROIs. To show the approximate location of individually-defined ROIs on the group template brain (Fig. 2D), we projected the individual ROI masks to the group template using spherical normalization and computed probability maps across subjects/hemispheres for each ROI. We defined the corresponding 
group ROIs as the regions where the probability for a given ROI was both larger than for any other ROI and larger than $50 \%$.

\section{Estimation of ROl-average response and adaptation tuning widths}

ROI-average response tuning widths were derived similarly to the smoothed local response tuning widths, by first recentering the voxelwise response tuning curves to their bias-corrected centroid frequencies, averaging them across all voxels within a given ROI, and, finally, fitting them with Gaussian-shaped functions (on the ERB-number scale) to estimate the function scale (peak response) and spread.

To derive the ROI-average adaptation tuning widths, we first estimated the adapted response to the probe when it was preceded with different adaptor frequencies (P|A). This was done by taking the difference, AP - A, between the ROI-averaged responses to each adaptorprobe pair, AP, and adaptor-only stimulus, A, at the corresponding adaptor frequency. We then calculated the amount of adaptation for each adaptor frequency by subtracting the adapted probe responses, $\mathrm{P} \mid \mathrm{A}$, from the unadapted ROI-averaged response to the probeonly stimulus, P. The resulting differences were expressed as a proportion of the ROI-averaged probe-only response $[(P-P \mid A) / P]$. To measure the widths of the ROl-average adaptation tuning curves (thought to represent the average neuronal tuning width), we fitted them with two types of Gaussian functions. For the first fit, we assumed that adaptation was entirely stimulus-specific (i.e., specific to the probe frequency) and would thus approach zero for sufficiently remote adaptor frequencies. Thus, the fitted functions were simple Gaussians on the ERB-number scale, with free spread and scale parameters, representing tuning width and maximum adaptation amount (when adaptor and probe frequencies matched), respectively. However, in most ROls, adaptation seemed to approach a non-zero floor, suggesting that a proportion of the adaptation effect was stimulus-independent. Thus, for the second fit, we fitted Gaussians with a constant offset as an additional free parameter. As ROI-average adaptation was always greatest when the adaptor frequency matched the probe frequency (see Fig. 4B), the location of the function mode was fixed at the probe frequency for both fits.

Gaussian functions were fitted both individually to each participant's hemisphere- and ROIspecific tuning curves (individual fits) and also to the corresponding average tuning curves across all 22 hemispheres (group fits). Confidence intervals for the group-fitted functions and parameters were obtained by bootstrap resampling the 22 hemispheres (with replacement) and fitting each sample average tuning curve in the same way as the group-average curve. Differences in the fitting parameters between ROls were tested with a linear mixed-effect model ( $R$ version 4.0.3, function Imer in package afex), using the individually fitted parameters after rejection of outliers in the spread parameter using the median absolute deviation method. Post-hoc pairwise comparisons were adjusted for family-wise error rate using Tukey's 
range test ( $R$ function Ismeans in package emmeans). All tuning spread parameter estimates are reported as FWHM of the best-fitting Gaussian functions. Goodness of fit and model selection indicators $\left(r^{2}\right.$, corrected Akaike information criterion, AICc, and Bayes information criterion, $\mathrm{BIC}$ ) were computed with respect to the individual hemisphere tuning curves.

In addition to the ROI-average response and adaptation tuning curves, we computed groupaverage maps of the adaptor and probe responses (GLM parameter estimates), and of the unnormalized and normalized adaptation effects $[(P-P \mid A)$ and $(P-P \mid A) / P]$. For this, the spherically-normalized individual responses and adaptation effects were first averaged across all cortical depths and then smoothed along the flattened cortical surface with a Gaussian kernel (FWHM=6 mm). The group-average maps were tested for statistical significance against zero using mass univariate one-sample $\dagger$ tests, corrected for multiple tests across all voxels of the left-hemisphere flattened cortical template using an adaptive FDR correction method [34].

\section{Computational model of fMRI adaptation in tonotopically-organized cortex}

To gain a better understanding of how fMRI-based response and adaptation tuning widths are related to the underlying neuronal tuning widths, we compared the measured data with simulations based on a computational model of $\mathrm{fMRI}$ adaptation. The model implemented an array of approximately hundred $1.5 \mathrm{~mm}$ unidimensional voxels sampling a unidimensional tonotopically-organized cortical strip containing a population of frequency-selective neurons (the exact number of voxels depended on the value of the tonotopic gradient, see below). We used this model to predict the amplitude of the BOLD responses (both at the individual voxel level and averaged across the array) to the adaptor-only (A), probe-only (P) and adaptor-probe (AP) stimuli, (ignoring the temporal dimension of the stimuli and responses for simplicity).

Neurons had Gaussian-shaped, unit-scaled response tuning curves to narrowband stimuli ( A and P), $R_{\text {cell }}\left(f \mid f_{c}, \sigma\right)=\exp \left(-\left(f_{c}-f\right)^{2} /\left(2 \sigma^{2}\right)\right)$, where $f$ is the stimulus frequency on the ERBnumber scale, $f_{c}$ is the neuron's characteristic frequency and $\sigma$ determines its tuning width (in ERBs). $f_{c}$ was limited to the range of human hearing between 0.02 and $18 \mathrm{kHz}$. Neuronal adaptation to an adaptor was modelled by decreasing the unadapted response to the probe, $R_{\text {cell }}(P)=R_{\text {cell }}\left(f_{P} \mid f_{c}, \sigma\right)$, multiplicatively by an amount proportional to the adaptor response, $R_{\text {cell }}(A)=R_{\text {cell }}\left(f_{A} \mid f_{c}, \sigma\right)$ :

$$
R_{\text {cell }}(P \mid A)=R_{\text {cell }}(P) \times\left[1-\kappa \times R_{\text {cell }}(A)\right]
$$

with proportionality constant, $\kappa$, representing the maximum adaptation strength (i.e. the maximum adaptation that would occur when the adaptor frequency, $f_{A}$, matches the neuron's characteristic frequency, $f_{c}$, and therefore $R_{\text {cell }}(A)=1$ ). Both parameters $\sigma$ and $\kappa$ were assumed to be constant across the entire neuron population. 
Neuronal characteristic frequencies, $f_{c}$, were tonotopically organized, increasing, on average, from one end of the cortical strip to the other. To allow for tonotopic scatter, the actual characteristic frequencies $\left(f_{c}\right)$ at each point in the cortical strip were allowed to vary randomly around a nominal value, $\varphi$, which increased linearly in ERB-numbers per millimetre to create a given tonotopic gradient (e.g., 2.2 ERB/mm). Thus, at any point along the strip, $f_{c}$ was allowed to vary according to a truncated Gaussian distribution centred on the local value of $\varphi$ :

$$
\theta\left(f_{c} \mid \varphi, \delta\right)=\frac{\exp \left(-\left(\varphi-f_{c}\right)^{2} /\left(2 \delta^{2}\right)\right)}{\sqrt{2 \pi} \times \delta}, 0.05<f_{c}<18 \mathrm{kHz}
$$

The spread parameter, $\delta$, of the scatter distribution controls the degree of randomness in local characteristic frequency values, with zero scatter $(\delta=0)$ corresponding to a perfectly ordered spatial progression of characteristic frequencies, and infinite scatter $(\delta=\infty)$ corresponding to a completely random distribution of characteristic frequencies across the strip. Note that changing the amount of scatter also changed the slope of the average characteristic frequency progression, or tonotopic gradient, across the strip.

The neuron population was sampled using an array of equally-spaced, but overlapping, 1.5 $\mathrm{mm}$ voxels. Although voxels overlapped, they were spaced in such a way that there was an integer number of contiguous voxels spanning a range of nominal characteristic frequencies $(\varphi)$ between about 0.05 and $16 \mathrm{kHz}$. The exact range was adjusted as required to create a given tonotopic gradient (e.g., 2.2 ERB/mm).

The BOLD response to a given stimulus ( $A, P$ or $A P$ ) in a given voxel was obtained by, first, integrating the neuronal responses to the stimulus across the full distribution of characteristic frequencies, $f_{c}$, of neurons contained within the voxel, then applying a non-linearity to account for BOLD refractoriness [e.g., 35, 36] and, finally, convolving with a hemodynamic point spread function (PSF).

The average neuronal response in a voxel (i.e. before applying the non-linearity and convolving with the PSF), $R_{\text {voxel }}(f)$, to an A or P stimulus centered at frequency $f$ depended on the distribution of characteristic frequencies within the voxel, and therefore on both the value of the spatial gradient of $\varphi$ and on the scatter parameter $\delta$ :

$$
R_{\text {voxel }}(f)=\int_{\varphi_{1}}^{\varphi_{2}} \int_{f_{1}}^{f_{2}} R_{\text {cell }}\left(f \mid f_{c}, \sigma\right) \times \theta\left(f_{c} \mid \varphi, \delta\right) d f_{c} d \varphi
$$

where $\varphi_{1}$ and $\varphi_{2}$ are the lower and upper bounds of the nominal characteristic frequencies within the voxel, as determined by the tonotopic gradient, and $f_{1}$ and $f_{2}$ are the bounds of the hearing range (0.01-18 kHz), all expressed in ERBs. 
A voxel's average adapted neuronal response to the probe (i.e., following an adaptor stimulus) was similarly computed by integrating the adapted neuronal responses $R_{\text {cell }}(P \mid A)$ within the voxel:

$$
R_{\text {voxel }}(P \mid A)=\int_{\varphi_{1}}^{\varphi_{2}} \int_{f_{1}}^{f_{2}} R_{\text {cell }}\left(f_{P} \mid f_{c}, \sigma\right) \times\left[1-\kappa_{\text {cell }} \times R_{\text {cell }}\left(f_{A} \mid f_{c}, \sigma\right)\right] \times \theta\left(f_{c} \mid \varphi, \delta\right) d f_{c} d \varphi
$$

Finally, the voxel's average neuronal response to an adaptor-probe pair, $R_{\text {voxel }}(A P)$, was computed as the sum of $R_{\text {voxel }}(A)$ and $R_{\text {voxel }}(P \mid A)$.

The BOLD non-linearity was modelled as a compressive function, $s$, applied to the neuronal voxel response of each type of stimulus (A, P or AP) and implemented as the positive half of a re-centered logistic function, such that $S(0)=0$, and scaled to have unit derivative at 0 and to reach a saturated BOLD level $\gamma$ :

$$
s(x)=\gamma\left(\frac{2}{1+\exp \left(\frac{-2 x}{\gamma}\right)}-1\right)
$$

The hemodynamic PSF was modelled by a spatial Gaussian convolution kernel, $g$, with an FWHM of $2 \mathrm{~mm}$. Thus, a voxel's BOLD response to a given stimulus, $\mathrm{S}$, was given by $R_{B O L D}(S)=$ $g \circ S\left(R_{\text {voxel }}(S)\right)$. To avoid edge effects when convolving responses with the hemodynamic PSF (see below), we added mirror images of the cortical strip and voxel array on each side of it (i.e. with characteristic frequency progressions opposite to that in the main array, resulting in frequency reversals). Responses from voxels in these duplicated arrays were otherwise ignored.

In actual experimental conditions, the BOLD response to the adapted probe (corresponding to $R^{\prime \prime}{ }_{\text {voxel }}(P \mid A)$ in the model), cannot be observed on its own, because, due to the temporal sluggishness of the hemodynamic response, it overlaps with the response to the preceding adaptor (corresponding to $R_{B O L D}(A)$ ). For comparison with the experimental fMRI data, we thus estimated $R_{B O L D}(P \mid A)$ by calculating the difference between the responses to the respective adaptor probe pair and adaptor-alone stimuli, $\widehat{R}_{B O L D}(P \mid A)=R_{B O L D}(A P)-R_{B O L D}(A)$. The associated adaptation effect was then obtained by calculating the difference between the unadapted and adapted probe responses, $R_{B O L D}(P)-\hat{R}_{B O L D}(P \mid A)$, as in the experiment.

The modelled voxelwise adaptor-only BOLD responses and BOLD adaptation effects at different adaptor frequencies were then used to construct array-average BOLD responses and $B O L D$ adaption tuning curves and derive the associated tuning widths. This was done in exactly the same way as for the corresponding experimental data. In particular, to create arrayaverage response tuning curves (based on the adaptor-only responses), the modelled voxelwise response tuning curves were first recentered on their estimated bias-corrected centroids. Tuning widths were then derived by fitting the tuning curves with Gaussian functions 
and the adaptation tuning curves were fitted twice - with or without an added constant offset to represent any stimulus-independent adaptation.

\section{Exploring and fitting the model}

We first explored the model's behavior informally by manually setting its four free parameters (neuronal tuning spread, $\sigma$, tonotopic scatter, $\delta$, neuronal adaptation strength, $\kappa$, BOLD saturation point, $\gamma$ ) to specific values (see Fig. 6). The nominal neuronal characteristic frequency gradient was set to $2.2 \mathrm{ERBs} / \mathrm{mm}$, close to the tonotopic gradient measured in our data (see below). To obtain smooth-looking tuning curves, we simulated responses for 50 adaptor frequencies ranging between 0.19 and $7.83 \mathrm{kHz}$, rather than just the seven frequencies used in the experiment.

Then, we fitted the model parameters by minimizing the sum of squared differences between the measured group-average and ROl-average response and adaptation tuning curves and the corresponding array-average model tuning curves (with each ROI modelled as a separate tonotopic array), simulated for the seven adaptor frequencies and five adaptor-probe pairs used in the experiment. Response and adaptation tuning curves from the two gradient and the two belt ROls were fitted jointly using the constrained non-linear programming solver fmincon in Matlab (with the default interior-point algorithm). During fitting, both the modelled and measured tuning curves were normalized to the peak of the response tuning curve. This was to avoid having to include a scaling parameter to match the (arbitrary) units of the modelled responses to those of the measured ones. The response tuning curves contained a maximum of 17 data points (due to recentering voxel tuning curves according to their biascorrected centroid) and the adaptation tuning curves contained five points, yielding a total of 22 data points for each ROI. To equate the contribution of each type of tuning curve (response versus adaptation) to the fit, the cost function (sum of squared errors) was weighted inversely to the tuning curve area (given by the sum of all its data points). In each fit, the neuronal tuning spread, $\sigma$, the tonotopic scatter, $\delta$, and the neuronal adaptation strength, $\kappa$, were allowed to vary between ROls, whilst the BOLD saturation level, $\gamma$, was fixed across ROls, resulting in a total of $10(3 \times 3+1)$ free parameters for each fit. $\sigma, \delta$ and $\gamma$ were constrained to be positive and $\kappa$ was constrained to range between 0 and 1 . The model's nominal tonotopic gradient was set to the gradient observed in the actual data in each ROI, averaged across the 22 hemispheres. The gradient was computed in each ROI of each individual hemisphere by averaging the local preferred-frequency (bias-corrected centroid, see above) gradient measured at each face of the 3D cortical mesh [37] contained in each ROI.

The model was fitted 1000 times in order to obtain the distributions and joint distributions of best-fitting parameter values. For each fit, the initial parameter values were drawn randomly from predefined intervals ([0 50] ERBs for $\sigma$ and $\delta,[01]$ for $\kappa$, and [ $1+\infty]$ for $\gamma$ ). The interval for $\gamma$ 
corresponded to a BOLD saturation point between 1.79 and $+\infty \%$ signal change when taking into account the tuning curve normalization constant averaged across ROIs).

\section{Comparisons with tuning width estimates from the human and non- human primate electrophysiological literature}

To compare our fMRI-adaptation-based frequency tuning width estimates with neuronal tuning widths measured electrophysiologically, we digitized data from seven studies that reported single- or multi-unit excitatory frequency tuning width from auditory neurons in core and/or belt fields using different non-human primate species in either anesthesized or unanesthesized recording conditions [see supplementary Table 1; 1, 38-43], and from 3 EEG studies that used a frequency adaptation paradigm similar to ours and measured adaptation tuning of the N1 and/or P2 ERP components $[17,18,44]$.

To make the non-human primate measurements as comparable to our fMRI-based tuning width estimates as possible, we converted the digitized primate tuning widths, originally given either as iso-response quality factors or as iso-response tuning widths in octaves, to iso-intensity FWHMs based on an assumed Gaussian tuning shape on the human ERB-number scale (see supplementary methods section for details).

Digitized frequency adaptation data from the three EEG studies were re-expressed as relative decrease in the unadapted $N 1$ response amplitude (or N1/P2 amplitude difference) to a probe presented alone, when this probe was preceded by adaptors at different frequencies (normalized adaptation in \% as in our Fig. 3F and $4 \mathrm{~B}$ ), and the adaptor-probe frequency difference was re-expressed in cochlear ERBs. The resulting adaptation tuning curves (shown in supplementary Fig. 2C) were fitted with simple Gaussian functions centered on the probe frequency to obtain the tuning FWHM.

\section{Funding}

This work was supported by the UK Medical Research Council (G0901321, MC_U135097128, MC_UU_00010/2) and the American University of Beirut Research Board.

\section{References}

1. Bartlett EL, Sadagopan S, Wang X. Fine frequency tuning in monkey auditory cortex and thalamus. J Neurophysiol. 2011;106(2):849-59.

2. Bitterman $Y$, Mukamel R, Malach R, Fried I, Nelken I. Ultra-fine frequency tuning revealed in single neurons of human auditory cortex. Nature. 2008;451 (7175):197-201.

3. Shera CA, Guinan JJ, Jr., Oxenham AJ. Revised estimates of human cochlear tuning from otoacoustic and behavioral measurements. Proc Natl Acad Sci U S A. 2002;99(5):3318-23. 
4. Besle J, Mougin O, Sanchez-Panchuelo RM, Lanting C, Gowland P, Bowtell R, et al. Is human auditory cortex organization compatible with the monkey model? Contrary evidence from ultra-high-field functional and structural MRI. Cereb cortex. 2019;29(1):41028.

5. Thomas JM, Huber E, Stecker GC, Boynton GM, Saenz M, Fine I. Population receptive field estimates of human auditory cortex. Neuroimage. 2015;105:428-39.

6. De Martino F, Moerel M, XU J, van de Moortele PF, Ugurbil K, Goebel R, et al. HighResolution Mapping of Myeloarchitecture In Vivo: Localization of Auditory Areas in the Human Brain. Cereb cortex. 2015;25(10):3394-405.

7. Moerel M, De Martino F, Formisano E. Processing of natural sounds in human auditory cortex: tonotopy, spectral tuning, and relation to voice sensitivity. J Neurosci. 2012;32(41):14205-16.

8. Collins CE, Turner EC, Sawyer EK, Reed JL, Young NA, Flaherty DK, et al. Cortical cell and neuron density estimates in one chimpanzee hemisphere. Proc Natl Acad Sci U S A. 2016;1 13(3):740-5.

9. Collins CE, Airey DC, Young NA, Leitch DB, Kaas JH. Neuron densities vary across and within cortical areas in primates. Proc Natl Acad Sci U S A. 2010;107(36):15927-32.

10. Shmuel A, Yacoub E, Chaimow D, Logothetis NK, Ugurbil K. Spatio-temporal point-spread function of fMRI signal in human gray matter at 7 Tesla. Neuroimage. 2007;35(2):539-52.

11. Blakemore C, Campbell FW. On the existence of neurones in the human visual system selectively sensitive to the orientation and size of retinal images. J Physiol. 1969;203(1):23760.

12. Barron HC, Garvert MM, Behrens TE. Repetition suppression: a means to index neural representations using BOLD? Philos Trans R Soc Lond B Biol Sci. 2016;371 (1705).

13. Grill-Spector K, Henson R, Martin A. Repetition and the brain: neural models of stimulusspecific effects. Trends Cogn Sci. 2006;10(1):14-23.

14. Tootell RB, Hadjikhani NK, Vanduffel W, Liu AK, Mendola JD, Sereno MI, et al. Functional analysis of primary visual cortex (V1) in humans. Proc Natl Acad Sci U S A. 1998;95(3):8117.

15. Fang F, Murray SO, Kersten D, He S. Orientation-tuned FMRI adaptation in human visual cortex. J Neurophysiol. 2005;94(6):4188-95.

16. Briley PM, Krumbholz K. The specificity of stimulus-specific adaptation in human auditory cortex increases with repeated exposure to the adapting stimulus. J Neurophysiol. 2013;110(12):2679-88.

17. Picton TW, Woods DL, Proulx GB. Human auditory sustained potentials. II. Stimulus relationships. Electroencephalogr Clin Neurophysiol. 1978;45(2):198-210.

18. Butler RA. Effect of changes in stimulus frequency and intensity on habituation of the human vertex potential. J Acoust Soc Am. 1968;44(4):945-50. 
19. Herrmann B, Henry MJ, Scharinger M, Obleser J. Auditory filter width affects response magnitude but not frequency specificity in auditory cortex. Hear Res. 2013;304:128-36.

20. Herrmann B, Henry MJ, Obleser J. Frequency-specific adaptation in human auditory cortex depends on the spectral variance in the acoustic stimulation. J Neurophysiol. 2013;109(8):2086-96.

21. Näätänen R, Sams M, Alho K, Paavilainen P, Reinikainen K, Sokolov EN. Frequency and location specificity of the human vertex N1 wave. Electroencephalogr Clin Neurophysiol. 1988;69(6):523-31.

22. Picton TW, Alain C, Woods DL, John MS, Scherg M, Valdes-Sosa P, et al. Intracerebral sources of human auditory-evoked potentials. Audiol Neurootol. 1999;4(2):64-79.

23. Yvert B, Fischer C, Bertrand $O$, Pernier J. Localization of human supratemporal auditory areas from intracerebral auditory evoked potentials using distributed source models. Neuroimage. 2005;28(1):140-53.

24. Larsson J, Solomon SG, Kohn A. fMRI adaptation revisited. Cortex. 2016:80:154-60.

25. Glasberg BR, Moore BC. Derivation of auditory filter shapes from notched-noise data. Hear Res. 1990;47(1-2):103-38.

26. Moore BC, Glasberg BR. Suggested formulae for calculating auditory-filter bandwidths and excitation patterns. J Acoust Soc Am. 1983;74(3):750-3.

27. Greenwood DD. A cochlear frequency-position function for several species--29 years later. J Acoust Soc Am. 1990;87(6):2592-605.

28. Glasberg BR, Moore BC. Frequency selectivity as a function of level and frequency measured with uniformly exciting notched noise. J Acoust Soc Am. 2000;108(5 Pt 1):231828.

29. Hawkins JE, Stevens SS. The Masking of Pure Tones and of Speech by White Noise. The Journal of the Acoustical Society of America. 1950;22(1):6-13.

30. Dale AM, Fischl B, Sereno MI. Cortical surface-based analysis. I. Segmentation and surface reconstruction. Neuroimage. 1999;9(2):179-94.

31. Fischl B, Sereno MI, Tootell RB, Dale AM. High-resolution intersubject averaging and a coordinate system for the cortical surface. Hum Brain Mapp. 1999;8(4):272-84.

32. Gardner JL, Merriam EP, Schluppeck D, Besle J, Heeger DJ. mrTools: Analysis and visualization package for functional magnetic resonance imaging data. . 2018.

33. Schönwiesner M, Dechent P, Voit D, Petkov Cl, Krumbholz K. Parcellation of Human and Monkey Core Auditory Cortex with fMRI Pattern Classification and Objective Detection of Tonotopic Gradient Reversals. Cereb cortex. 2015;25(10):3278-89

34. Benjamini Y, Krieger AM, Yekutieli D. Adaptive linear step-up procedures that control the false discovery rate. Biometrika. 2006:93(3):491-507.

35. Inan S, Mitchell T, Song A, Bizzell J, Belger A. Hemodynamic correlates of stimulus repetition in the visual and auditory cortices: an fMRI study. Neuroimage. 2004;21 (3):886-93. 
36. Kumar S, Penny W. Estimating neural response functions from fMRI. Front Neuroinform. 2014:8:48.

37. Mancinelli C, Livesu M, Puppo E. A comparison of methods for gradient field estimation on simplicial meshes. Computers \& Graphics-Uk. 2019;80:37-50.

38. Cheung SW, Bedenbaugh PH, Nagarajan SS, Schreiner CE. Functional organization of squirrel monkey primary auditory cortex: responses to pure tones. J Neurophysiol. 2001;85(4):1732-49.

39. Kajikawa $Y$, de La Mothe L, Blumell S, Hackett TA. A comparison of neuron response properties in areas $\mathrm{Al}$ and $\mathrm{CM}$ of the marmoset monkey auditory cortex: tones and broadband noise. J Neurophysiol. 2005;93(1):22-34.

40. Pelleg-Toiba R, Wollberg Z. Tuning properties of auditory cortex cells in the awake squirrel monkey. Exp Brain Res. 1989;74(2):353-64.

41. Philibert B, Beitel RE, Nagarajan SS, Bonham BH, Schreiner CE, Cheung SW. Functional organization and hemispheric comparison of primary auditory cortex in the common marmoset (Callithrix jacchus). J Comp Neurol. 2005;487 (4):391-406.

42. Recanzone GH, Guard DC, Phan ML. Frequency and intensity response properties of single neurons in the auditory cortex of the behaving macaque monkey. J Neurophysiol. 2000;83(4):2315-31.

43. Recanzone GH, Schreiner CE, Merzenich MM. Plasticity in the frequency representation of primary auditory cortex following discrimination training in adult owl monkeys. J Neurosci. 1993;13(1):87-103.

44. Briley PM, Breakey C, Krumbholz K. Evidence for pitch chroma mapping in human auditory cortex. Cereb cortex. 2013;23(11):2601-10.

45. Merzenich MM, Brugee JF. Representation of the cochlear partition on the superior temporal plane of the macaque monkey. Brain Res. 1973;50:275-96.

46. Lee HA, Lee SH. Hierarchy of direction-tuned motion adaptation in human visual cortex. J Neurophysiol. 2012;107(8):2163-84.

47. Grill-Spector K, Malach R. fMR-adaptation: a tool for studying the functional properties of human cortical neurons. Acta Psychol (Amst). 2001;107(1-3):293-321.

48. Weigelt S, Limbach K, Singer W, Kohler A. Orientation-selective functional magnetic resonance imaging adaptation in primary visual cortex revisited. Hum Brain Mapp. 2012;33(3):707-14.

49. Heckman GM, Bouvier SE, Carr VA, Harley EM, Cardinal KS, Engel SA. Nonlinearities in rapid event-related fMRI explained by stimulus scaling. Neuroimage. 2007;34(2):651-60.

50. Zhou J, Benson NC, Kay KN, Winawer J. Compressive Temporal Summation in Human Visual Cortex. J Neurosci. 2018;38(3):691-709.

51. Zhang $\mathrm{N}$, Zhu XH, Chen W. Investigating the source of BOLD nonlinearity in human visual cortex in response to paired visual stimuli. Neuroimage. 2008;43(2):204-12. 
52. Pihlaja M, Henriksson L, James AC, Vanni S. Quantitative multifocal fMRI shows active suppression in human V1. Hum Brain Mapp. 2008;29(9):1001-14.

53. Kosaki H, Hashikawa T, He J, Jones EG. Tonotopic organization of auditory cortical fields delineated by parvalbumin immunoreactivity in macaque monkeys. J Comp Neurol. 1997;386(2):304-16.

54. Morel A, Garraghty PE, Kaas JH. Tonotopic organization, architectonic fields, and connections of auditory cortex in macaque monkeys. J Comp Neurol. 1993;335(3):437-59.

55. Moshitch D, Las L, Ulanovsky N, Bar-Yosef $O$, Nelken I. Responses of neurons in primary auditory cortex (A1) to pure tones in the halothane-anesthetized cat. J Neurophysiol. 2006:95(6):3756-69.

56. Bartels A, Logothetis NK, Moutoussis K. fMRI and its interpretations: an illustration on directional selectivity in area V5/MT. Trends Neurosci. 2008;31 (9):444-53.

57. Goense JB, Logothetis NK. Neurophysiology of the BOLD fMRI signal in awake monkeys. Curr Biol. 2008;18(9):631-40.

58. Xie R, Gittelman JX, Pollak GD. Rethinking tuning: in vivo whole-cell recordings of the inferior colliculus in awake bats. J Neurosci. 2007;27(35):9469-81.

59. Buxton RB. Dynamic models of BOLD contrast. Neuroimage. 2012;62(2):953-61.

60. Rothschild G, Nelken I, Mizrahi A. Functional organization and population dynamics in the mouse primary auditory cortex. Nat Neurosci. 2010;13(3):353-60.

61. Bandyopadhyay S, Shamma SA, Kanold PO. Dichotomy of functional organization in the mouse auditory cortex. Nat Neurosci. 2010;13(3):361-8.

62. Carandini $M$, Ferster D. A tonic hyperpolarization underlying contrast adaptation in cat visual cortex. Science. 1997;276(5314):949-52.

63. Sawamura H, Orban GA, Vogels R. Selectivity of neuronal adaptation does not match response selectivity: a single-cell study of the FMRI adaptation paradigm. Neuron. 2006;49(2):307-18.

64. Liu Y, Murray SO, Jagadeesh B. Time course and stimulus dependence of repetitioninduced response suppression in inferotemporal cortex. J Neurophysiol. 2009;101 (1):41836.

65. De Baene W, Vogels R. Effects of adaptation on the stimulus selectivity of macaque inferior temporal spiking activity and local field potentials. Cereb cortex. 2010;20(9):21 45-65.

66. Abbott LF, Varela JA, Sen K, Nelson SB. Synaptic depression and cortical gain control. Science. 1997;275(5297):220-4.

67. Alink A, Abdulrahman H, Henson RN. Forward models demonstrate that repetition suppression is best modelled by local neural scaling. Nat Commun. 2018:9(1):3854.

68. Brosch $\mathrm{M}$, Scheich $\mathrm{H}$. Tone-sequence analysis in the auditory cortex of awake macaque monkeys. Exp Brain Res. 2008;184(3):349-61. 
69. Scholes C, Palmer AR, Sumner CJ. Forward suppression in the auditory cortex is frequencyspecific. Eur J Neurosci. 2011;33(7):1240-51.

70. Reyes AD. Synaptic short-term plasticity in auditory cortical circuits. Hear Res. 2011;279(12):60-6.

71. Wehr M, Zador AM. Synaptic mechanisms of forward suppression in rat auditory cortex. Neuron. 2005;47(3):437-45.

72. Brosch M, Schulz A, Scheich H. Processing of sound sequences in macaque auditory cortex: response enhancement. J Neurophysiol. 1999:82(3):1542-59.

73. Solomon SG, Kohn A. Moving sensory adaptation beyond suppressive effects in single neurons. Curr Biol. 2014;24(20):R1012-22.

74. Mill R, Coath M, Wennekers T, Denham SL. A neurocomputational model of stimulusspecific adaptation to oddball and Markov sequences. PLoS Comput Biol. $2011 ; 7(8): e 1002117$.

75. Vogels R. Sources of adaptation of inferior temporal cortical responses. Cortex. 2016;80:185-95. 